\title{
Effective diffusivity in partially-saturated carbon-fiber gas diffusion layers: Effect of through-plane saturation distribution
}

\author{
Pablo A. García-Salaberri ${ }^{1}$, Gisuk Hwang ${ }^{2}$, Marcos Vera ${ }^{1}$, Adam Z. Weber ${ }^{3}$ \\ and Jeff T. Gostick ${ }^{4, A}$ \\ ${ }^{1}$ Dept. de Ingeniería Térmica y de Fluidos, Universidad Carlos III de Madrid, Leganés, \\ 28911, Spain \\ ${ }^{2}$ Dept. of Mechanical Engineering, Wichita State University, Wichita, KS, USA \\ ${ }^{3}$ Environmental Energy Technologies Division, Lawrence Berkeley National Laboratory, \\ Berkeley, CA, USA \\ ${ }^{4}$ Dept. of Chemical Engineering, McGill University, Montreal, QC, Canada

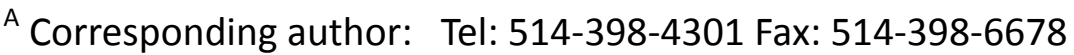 \\ E-mail: jeff.gostick@mcgill.ca \\ URL: http://pmeal.com/
}




\section{Abstract}

The effective diffusivity of gaseous species in partially-saturated finite-size porous media is a valuable parameter for mathematical modeling of many processes, but it is difficult to measure experimentally. In this work, the effective diffusivity of carbon-fiber gas diffusion layers (GDLs) used in polymer electrolyte fuel cells (PEFCs) was determined by performing lattice Boltzmann (LB) simulations on X-ray tomographic reconstructions of invading water configurations. Calculations on dry GDLs were in close agreement with previous experimental data; the effective diffusivity was reduced by the addition of PTFE due to the loss of pore volume and the higher tortuosity of transport paths. The effect of water saturation was significantly larger. It was found that the resistance of water to gas transport was extremely dependent on the saturation distribution through the porous medium, particularly the peak saturation, and not just the average saturation as is typically considered in the literature. Through-plane diffusion was dramatically limited in materials with high-peak local saturations, even at low average saturation levels. No significant limitations were observed for diffusion in the material plane. The computed results demonstrate the strong sensitivity of finite-size porous media to local conditions, highlighting the difficulties of applying volume-averaged continuum-scale modeling techniques to micro-scale materials.

Keywords: effective diffusivity; gas diffusion layer; water saturation; finite size; X-ray tomography; lattice Boltzmann method 


\section{Introduction}

Polymer electrolyte fuel cells (PEFCs) are a leading candidate to replace internal combustion engines for automotive applications, as they are the only presently available technology that can offer equivalent power density, range and refueling times in a sustainable way. The electrochemical conversion of reactants in PEFCs leads to virtually zero emissions of pollutants (water and heat are the only products generated in the cell), and provides 2-3 times higher efficiency as compared to high-temperature combustion devices [1]. However, engineering factors such as high cost and limited durability remain significant barriers to large-scale commercialization. To reduce cost, it is imperative that the mass of platinum (Pt) catalyst per vehicle be reduced significantly; this can be achieved by lowering the loading of Pt per unit active cell area, or reducing the active area (or both). The latter approach, however, requires an increase of the current density $\left(\mathrm{A} / \mathrm{m}^{2}\right)$ of the cells, to maintain the output power (W) of the stack. Therefore, thorough understanding of the transport of reactants and products to/from the catalyst sites in PEFCs is needed [2-5].

One key element of a fuel-cell electrode is the gas diffusion layer (GDL), which is a thin highly porous layer composed of carbon fibers. The GDL must fulfill several functions such as providing structural stability to the cell, and transport pathways for gaseous reactants and liquid water through its pore volume and electronic current and heat through its solid structure. A deep knowledge of the effects of liquid water on the gas diffusivity of GDLs is essential to achieving an increased PEFC performance [2-5].

The standard approach for modeling the effective diffusivity in porous materials is to modify the bulk diffusion coefficient, $D_{\text {bulk }}$, to get an effective diffusivity, $D^{\text {eff }}$, through the porous domain [6],

$$
D^{\mathrm{eff}}=D_{\mathrm{bulk}} \frac{\varepsilon_{\mathrm{V}}}{\tau}
$$

where $\varepsilon_{\mathrm{V}}$ is the void volume fraction of the porous medium, and $\tau$ is the so-called 
tortuosity, which accounts for the indirect route that gas molecules must follow to traverse the 'tortuous' pore space. In porous media whose pore structure is partially filled with liquid water, gas diffusion is further blocked since liquid offers essentially no gas flux. The configuration, distribution, and extent of water within the pore space have major impacts on the diffusion rates, above and beyond the pore-structure effects [7]. To differentiate the impacts of the pore/throat structure of the dry material from those of the invading liquid, Eq.(1) can be decomposed into two independent functions, one accounting for the effect of the unsaturated medium, $f(\varepsilon)$, and another for the relative effect of liquid in the pore space, $g(s)$ :

$$
\frac{D^{\text {eff,wet }}}{D_{\text {bulk }}}=\frac{D^{\text {eff,dry }}}{D_{\text {bulk }}} \frac{D^{\text {eff,wet }}}{D^{\text {eff,dry }}}=f(\varepsilon) g(s)
$$

where $\varepsilon$ is the porosity, $s$ is the liquid saturation (defined as the ratio of liquid volume to pore volume), and $D^{\text {eff,dry }}$ and $D^{\text {eff,wet }}$ are the effective diffusivities of the porous medium under dry, and partially-saturated (or wet) conditions, respectively. A review of previous studies attempting to determine $f(\varepsilon)$ and $g(s)$ for fuel-cell GDL materials is presented below.

Multiple experimental [8-19], numerical [20-22] and semi-analytical [23-28] works on the dry effective diffusivity function, $f(\varepsilon)$, for carbon-paper GDLs can be found in the literature. These investigations provide fundamental information about the effects of compression, the fabrics of the substrate, the addition of wet-proofing agents, and the incorporation of micro-porous layers (MPLs) on the effective gas diffusivity of GDLs. Good agreement is observed among different authors. All of them show that the effective diffusivity of many carbon-fiber papers is anisotropic, with higher values in the in-plane direction due to preferential alignment of the GDL fiber structure in the material plane [8]. Moreover, it has been generally found that the effective diffusivity is notably lower than predicted by most established and widely used models, such as Bruggeman's effective medium theory [29], or the random fiber model by Tomadakis \& Sotirchos [30]. The deviations from these idealized models are explained by the complex 
structure formed by fibers, binder, and PTFE in carbon-paper materials. For instance, the sintering processes of carbon-fiber GDLs typically results in a webbed region at fiber intersection points that offers little porosity but dramatically increases tortuosity.

In contrast, very few experimental measurements on the relative effective diffusivity, $g(s)$, of GDLs have been reported in the literature [10,31,32]. This is due to the difficulty of measuring species concentration and flux in GDLs with thicknesses between 100 and $400 \mu \mathrm{m}$, while simultaneously controlling the amount of water. For instance, Utaka et al. [31] used custom GDLs, which were 10-20 times thicker than those actually used in fuel cells, in order to achieve a detectable concentration gradient. Hence, a wide variety of numerical strategies have been adopted to determine 'experimentally inaccessible' properties such as the effective diffusivity. This includes pore network modeling [7,3336]; pore-scale CFD models using reconstructions of either artificial or tomographic images of partially-saturated GDLs [21,22,37]; inverse modeling techniques in which macroscopic CFD models are combined with experiments to determine the free parameters of prescribed laws for the effective diffusivity [38,39]; and semi-analytical fractal models [23]. In general, the results reported in all these works show a large disparity, particularly for the through-plane direction. A power law of the form:

$$
g(s)=(1-s)^{\mathrm{n}},
$$

was used by most of the authors to describe their results, with $\mathrm{n}$ ranging from about 2 to more than 5 for the through-plane direction, and $\mathrm{n} \approx 2$ for the in-plane direction.

The above literature review shows that additional effort is still needed to shed further light on the influence of water saturation on the effective diffusivity of carbon-paper GDLs. In particular, better understanding of the effects of the water distribution through the GDL on diffusive transport is of paramount importance for the proper operation of PEFCs. A better knowledge of the transport properties of partially-saturated GDLs will also help to improve the predictive capabilities of macroscopic continuum models which are extensively used in fuel cell design [40-42]. One way to accomplish this goal is by the 
combination of tomographic reconstructions of GDLs and numerical models that explore transport processes at the pore-scale level $(\sim 10-100 \mu \mathrm{m})$. For this purpose, the lattice Boltzmann method (LBM) is an excellent option due to its ability to use 3D image stacks directly as a mesh, rather than sophisticated meshing tools as in traditional finite element (FEM) or finite volume (FVM) methods [43]. An increasing number of LBM studies exploring the properties of woven and nonwoven fabrics can be found in the fuel cell literature (see, e.g., [44-49]).

In the present work, the effective gas diffusivity of dry and partially-saturated carbonpaper GDLs is examined by performing LB simulations on X-ray computed tomography (XCT) images of water-invasion experiments. The structure of the paper is as follows. In Section 2, the methodology used for the invasion experiments and the reconstruction of the image stacks is presented. In Section 3, the algorithm adopted for the segmentation of the three phases composing the porous media (solid, air and water) is described. This also includes a comprehensive analysis of the morphological properties of the GDLs, and the amount and distribution of water in the invasion experiments. In Section 4, the LBM model and the case studies examined in the work are presented. The computed results are discussed in Section 5, which also incorporates a detailed comparison with previous data. The effective diffusivity in both the through- and in-plane directions of the material are investigated due to the anisotropic nature of carbon-paper GDLs. Finally, the concluding remarks are given in Section 6. 


\section{Experimental}

\subsection{Materials}

Experiments were performed on Toray ${ }^{\circledR}$ TGP-H-120 carbon-paper GDLs (Fuel Cell Earth LLC, USA). Uncompressed samples with 0 and $10 \%$ by weight PTFE loading were investigated. This material is somewhat thicker than GDLs typically used in modern fuel cells ( 375 vs. $250 \mu \mathrm{m}$ ) but the thicker domain allowed more space for the invading water fingers to evolve before percolation. In subsequent LBM simulations, the effective diffusivities of both the untreated and PTFE-treated dry GDLs were examined, while only the PTFE-treated GDL was analyzed under partially-saturated conditions. The untreated sample was excluded from the calculations due to the difficulty to prevent leakage of water between the sample and the holder in the experiments.

\subsection{Collection of Tomographic Data}

Data were collected at Lawrence Berkeley National Lab's Advanced Light Source (ALS) synchrotron (Beamline 8.3.2). Tomographic images were produced by collecting a series of radiographs of a sample as it was rotated $180^{\circ}$ on an axis normal to the X-ray beam. Radiographs were obtained every $0.15^{\circ}$ for a total of 1080 images. The exposure time for each image was $100 \mathrm{~ms}$, leading to a scan time of less than 2 minutes. An X-ray energy of $35 \mathrm{keV}$ was used so that images could be reconstructed using the phase contrast information, rather than absorption contrast. This approach requires filtering the radiographs to enhance the phase contrast information and this was accomplished using the modified Bronnikov algorithm [50]. All image filtering and reconstructions

were performed using the commercial software Octopus ${ }^{\circledR}$ 8.3. Very clear images with low noise and consistent greyscale values across the reconstructed images were obtained, which was essential for the subsequent segmentation of the image stacks as outlined below in Section 3. The resolution of the camera combined with optical magnification allowed for a $1.33 \mu \mathrm{m}$ voxel size. 


\subsection{Water-Invasion Experiments}

Tomographic data sets of GDLs were taken at various levels of water saturation. The experimental set-up used for the water-invasion experiments is shown in Figure 1 with the assorted parts labeled. The neck of the holder where the GDL sample of $3.2 \mathrm{~mm}$ in diameter was located was reduced as small as possible to limit X-ray attenuation by the holder walls $(\approx 0.5 \mathrm{~mm}$ thick). Water invaded into the $\mathrm{GDL}$ from the bottom face through a PTFE pipe with a $1.5 \mathrm{~mm}$ inner diameter, while air was allowed to escape out the top face through a PTFE membrane. The hydrophobic membrane prevented liquid water from leaving the system, thereby enabling saturations above the breakthrough point to be reached. The saturation level was controlled by increasing the static head of water in the injection pipe, which was arranged in a u-tube configuration. Because air was always at atmospheric pressure, the capillary pressure was equal to $p_{\mathrm{c}}=p_{\mathrm{l}}-p_{\mathrm{g}}=$ $\rho_{\mathrm{w}} g h$. Liquid pressure was raised by $10 \mathrm{~cm}$ increments $(\approx 1 \mathrm{kPa})$, with a full tomographic data set collected at each capillary pressure level. Experiments were finished between 7 and $8 \mathrm{kPa}$ when the sample was almost fully saturated above the breakthrough pressure $\left(p_{\mathrm{c}} \approx 3-4 \mathrm{kPa}\right)[33,51,52]$. 


\section{Image Processing}

The reconstructed 3D stacks were segmented in order to differentiate the three phases composing the system, i.e., GDL solid structure (fibers, binder and PTFE), water and air. The segmentation of the images was performed using custom-made code in MATLAB ${ }^{\circledR}$, as well as relying heavily on the image processing software Fiji for more common operations. The data exchange between MATLAB ${ }^{\circledR}$ and Fiji was done through the publicdomain Java package MIJI [53]. A representative square domain of $2 \times 2 \mathrm{~mm}$ centered on the injection pipe was extracted from the collected circular samples of $3.2 \mathrm{~mm}$ in diameter. Some slices at the top and bottom edges of the GDL were removed due to local interferences of the membrane and the pipe with the rough surface of the GDL. The full thickness of the GDL domains considered for segmentation was $L_{z}=0.32 \mathrm{~mm}$ and $L_{z}=0.28 \mathrm{~mm}$ for the untreated and PTFE-treated samples, respectively. This resulted in about a $15 \%$ and a $25 \%$ loss of material as compared with the manufacturer's datasheet $\left(L_{\mathrm{z}, \mathrm{GDL}}=0.37 \mathrm{~mm}\right)$ [54]. In order to explore the effect of the surface region lost in the above domains on the dry effective diffusivity, an extra subsample of $0.8 \times 0.8 \times 0.37 \mathrm{~mm}$, free or interference artifacts, was carefully selected for each of the GDLs from the dry sample stacks.

\subsection{Segmentation Algorithm}

The main steps followed for the segmentation of the images are presented below. Further details on the segmentation algorithm, including information on the intensity histograms and several sensitivity analyses, can be found in the supplementary material to this paper. The segmentation of the stacks into the three respective phases was performed sequentially due to the low contrast between solid- and water-phase voxels in the reconstructed images (see Figure 2(a)). First, a base segmentation of the solid structure was performed by global thresholding the bimodal dry sample stack using Otsu's method [55]. The resulting binary mask was then multiplied by the grayscale wet 
sample stack, so that solid-phase voxels were identified as zero-intensity black voxels; the result is shown in Figure 2(b). Next, a 3D morphological dilation/contraction operation with a spherical element of 3 voxels was applied to correct the imperfect matching of the solid structure around that previously determined with Otsu's method [55]. Note that this operation could be applied on the dry or on any of the wet sample stacks leading to similar results (see supplementary material for results obtained on a dry sample). As shown in Figure 2(c), the 3D morphological operation resulted in a slight perturbation of the void volume, while including most of the interfacial solid-phase voxels not identified by Otsu's method [55]. The above two steps, Otsu's method [55] and the dilation/contraction operation, led to the final segmentation of the solid phase, so that the next problem became differentiating water from air. To this end, an edgepreserving bilateral filter was applied to remove the overlap of some darkened regions in water fingers with the low-intensity air phase. The high time and memory requirements of this filter were reduced using an in-house version of the fast downsampling/upsampling algorithm by Paris \& Durand [56]. As shown in Figure 2(d), the bilateral filtering step gave excellent results, significantly enhancing the contrast between water and air phases, and reducing intensity fluctuations within each phase. Finally, water-phase voxels were identified by manual thresholding of the now nonoverlapping wet sample histogram. The partial results from the segmentation of the solid structure of the GDL and water were combined, and the final segmented stack was obtained (see Figure 2(e)).

\subsection{Morphological Properties and Water Distribution}

Several analyses were performed to confirm the proper segmentation of the dry and wet samples, involving both global and local characteristics of the porous media. The properties examined in the dry samples were the pore size distribution, 1D throughplane porosity profiles and average porosity, whereas the variables studied in the wet samples were the capillary pressure curve and 1D saturation distributions along the 
through- and in-plane directions.

\subsubsection{Dry Samples: Pore Size Distribution, Porosity Profiles and Average Porosity}

The pore size distribution of the dry sample stacks was scanned using the heuristic morphological image opening (MIO) algorithm described by Gostick [33]. As shown in Figure 3(a), good agreement is found between the cumulative pore size distributions (average mercury saturation vs. pore diameter) computed by the MIO algorithm and previous experimental data $[14,57,58]$. This result confirms that pore/throat sizes, as well as connectivity of the pore space, were well represented in the segmented GDL stacks. The characteristic pore diameter, $d_{\mathrm{p}}^{\text {char }}$, of Toray ${ }^{\circledR}$ TGP-H-120 is between 20-35 $\mu \mathrm{m}$, and does not vary significantly with the PTFE loading [52]. The somewhat lower pore diameters predicted by the MIO simulations are explained by the inability of this simple algorithm to account for some key physical phenomena, such as coalescence of menisci and non-spherical invasion fronts [33]. Given that the range of pore diameters in the examined porous media is between $10-60 \mu \mathrm{m}$, the $1.33 \mu \mathrm{m} /$ voxel resolution of the image stacks provided a sufficiently fine mesh for subsequent LBM simulations.

Figure $3(\mathrm{~b})$ shows the $x y$-averaged porosity profiles along the through-plane direction, $\bar{\varepsilon}_{\mathrm{z}}$, of the complete set of dry GDL domains analyzed in this work (see case studies in Section 4.1). As can be seen, the computed porosity profiles are in good agreement with previous studies for Toray ${ }^{\circledR}$ carbon-paper GDLs [59-61]. A central core region (CR) can be identified with rather strong fluctuations of the porosity due to the arrangement of the solid structure of the GDL, surrounded by two highly porous surface regions (SR) in which the solid structure progressively vanishes. The addition of PTFE leads to a net decrease of the porosity in the core region, yet the undulations in porosity are similar to the untreated case $[51,59]$. The similar porosity distributions observed in all the domains indicate that no anomalous features were present in the segmented samples. The porosities of the untreated/PTFE-treated domains mostly excluding the surface region of the material are $\varepsilon_{\mathrm{avg}}=0.69 / 0.66$, while the porosities of the domains including the 
core and surface regions $(C R+S R)$ are notably higher, $\varepsilon_{\text {avg }}=0.75 / 0.7$. Similar values were previously reported for uncompressed and compressed Toray ${ }^{\circledast}$ TGP-H-120 GDLs with and without PTFE loading (see $[9,10,51,52,57,59,62]$ among others).

\subsubsection{Wet Samples: Capillary Pressure Curve and Saturation Distribution}

Figure 4 shows the capillary pressure curve (average water saturation vs. capillary pressure) obtained in the present invasion experiments for the 10\% PTFE-treated sample, along with the experimental data reported by Gostick et al. [52]. Even though the waterinvasion experiments conducted here were not specifically designed to measure this property, the results are in reasonable agreement with Gostick et al. [52]. The somewhat lower breakthrough pressures ( $3-4 \mathrm{kPa}$ vs. $5 \mathrm{kPa}$ ), and the larger saturations reached in the present experiments at capillary pressures higher than $2 \mathrm{kPa}$ are ascribed to (i) the small size of the samples used for the X-ray visualizations as compared to [52] $\left(d_{\mathrm{GDL}}=3.2\right.$ $\mathrm{mm}$ vs. $d_{\mathrm{GDL}}=19 \mathrm{~mm}$ ), and (ii) the different injection conditions used in this work (pipe smaller than GDL sample) as compared to [52] (full-face invasion). The nonrepresentativeness of small samples to characterize the water retention properties of GDLs was previously observed in the ex-situ experiments of Flückiger et al. [61]. Note also that an accurate comparison with experimental capillary pressure curves on GDL samples of similar dimensions was not feasible as evaporation would significantly affect the exceedingly small volumes of liquid water injected at each step.

The $x y$ - and $y z$-averaged saturation profiles obtained along the through-, $\bar{s}_{z}$, and in-plane, $\bar{s}_{\mathrm{x}}$, directions for a $0.8 \times 0.8 \mathrm{~mm} 10 \%$ PTFE-treated GDL domain extracted from the large segmented sample of $2 \times 2 \mathrm{~mm}$ are shown in Figure 5 . Only one representative direction is shown in the material plane, since the profiles in other in-plane directions are similar. As can be seen, strong changes in the local saturation level are observed across the GDL, while no significant variations are found in the material plane. The high-gradient saturation profiles through the GDL arise due to the capillary-dominated percolation 
process of water. Given that in the experiments the GDL region located on the injection pipe is invaded homogenously, very high saturation levels are reached in the open surface region of low capillary resistance close to the invasion face. The amount of water gradually decreases towards the opposite face as water fingers percolates through the pore/throat structure of the core region of the GDL. As shown in Figure 5(a), the through-plane saturation distribution at the breakthrough point ( $p_{\mathrm{c}} \approx 3-4 \mathrm{kPa}$ ) varies from almost fully-saturated conditions, $\bar{s}_{\mathrm{z}} \approx 1$, near the inlet surface region to $\bar{S}_{\mathrm{z}} \approx 0.2$ on the face adjacent to the PTFE membrane. Similar results were presented in previous pore network and ex-situ experimental studies (see $[33,61,63]$ among others). The high saturation profiles in the present invasion experiments may then resemble those in an operational fuel cell under severe flooding conditions due, e.g., to a poor water removal rate from the catalyst layer and/or the existence of interfacial gaps between the GDL and the catalyst layer. Besides, these high-gradient saturation profiles provided an opportunity to examine the impact that lower saturation variations through the GDL has on the effective diffusivity. This analysis was accomplished by progressively removing larger portions of the GDL adjacent to the injection pipe in the LBM simulations (see details in Section 4.1). 


\section{Lattice Boltzmann Method}

Gas diffusive transport at the sub-pore scale level in the GDLs was simulated by means of the lattice Boltzmann method $[64,65]$. The model was implemented using the built-in capabilities of the parallel open-source LB solver Palabos [66]. A 3D seven-speed D3Q7 lattice with the standard Bhatnagar-Gross-Krook (BGK) approximation for the collision operator [67] was adopted for the calculations. The governing equation of the model in index notation is given by:

$$
f_{\alpha}\left(\boldsymbol{x}_{\mathbf{i}}+\boldsymbol{c}_{\boldsymbol{\alpha}} \Delta t, t+\Delta t\right)-f_{\alpha}\left(\boldsymbol{x}_{\mathbf{i}}, t\right)=-\frac{f_{\alpha}\left(\boldsymbol{x}_{\boldsymbol{i}}, t\right)-f_{\alpha}^{\mathrm{eq}}\left(\boldsymbol{x}_{\mathbf{i}}, t\right)}{\tau} ; \alpha=0, \ldots, 6
$$

where $f_{\alpha}\left(\boldsymbol{x}_{\mathbf{i}}, t\right)$ are the particle distribution functions at location $\boldsymbol{x}_{\mathbf{i}}$ and time $t, \Delta t$ is the time step, $\tau$ is the dimensionless relaxation time, and $\boldsymbol{c}_{\boldsymbol{\alpha}}$ and $f_{\alpha}^{\text {eq }}$ are the velocities and equilibrium populations of the distribution functions $f_{\alpha}$. The time increment, $\Delta t$, and lattice spacing, $\Delta \boldsymbol{x}_{\mathbf{i}}=\boldsymbol{c}_{\boldsymbol{\alpha}} \Delta t$, were set to 1 , as was the relaxation time $\tau$. This value ensured the stability and accuracy of the numerical scheme [68]. The lattice velocities and equilibrium distributions for the D3Q7 model are:

$$
\boldsymbol{c}_{\boldsymbol{\alpha}}=\left\{\begin{array}{l}
(0,0,0) \\
( \pm 1,0,0),(0, \pm 1,0),(0,0, \pm 1) \\
\alpha \neq 0
\end{array} \quad f_{\alpha}^{\mathrm{eq}}=\left\{\begin{array}{ll}
\left(1-3 c_{\mathrm{s}}^{2}\right) C & \alpha=0 \\
\left(c_{\mathrm{s}}^{2} / 2\right) C & \alpha \neq 0
\end{array} \quad\right. \text { Eq.(5) }\right.
$$

where $C$ is the macroscopic concentration, and $c_{\mathrm{s}}=1 / 2$ is the lattice 'speed of sound'.

The relationship of the macroscopic concentration, $C$, the bulk diffusivity, $D_{\text {bulk, }}$ and the Fickian diffusion flux, $\boldsymbol{j}$, with the mesoscopic variables $\left(f_{\alpha}, \boldsymbol{c}_{\boldsymbol{\alpha}}\right.$ and $\left.\tau\right)$ is obtained through the Chapmann-Enskog asymptotic analysis of Eq.(4) [68,69]:

$$
C=\sum_{\alpha=0}^{6} f_{\alpha} ; \quad D_{\text {bulk }}=c_{\mathrm{s}}^{2}\left(\tau-\frac{1}{2}\right) ; \quad \boldsymbol{j}=\left(1-\frac{1}{2 \tau}\right) \sum_{\alpha=0}^{6} f_{\alpha} \boldsymbol{c}_{\boldsymbol{\alpha}}
$$

A sketch of the computational domain used for the LBM simulations is shown in Figure 6(a). In addition to the GDL domain, a block of 5 gas-phase voxels was padded at both sides of the GDL in the direction of interest to mimic the inlet/outlet conditions in an experimental setup. The effective diffusivities in both the through-plane direction and 
one (representative) in-plane direction were examined in this work. The system was excited by imposing a concentration difference between 1 and 0 at the outermost faces in the direction under study according to the formulation of Zou \& He [70]. On the remaining faces, the geometry was padded with solid walls. The set-up of the numerical domain for the LBM simulations was performed in $M A T L A B^{\circledR}$. The segmented GDL samples (with air labeled 0 , solid 1 , and water 2 ), were conveniently padded with the exterior walls (labeled 1) and inlet/outlet gas zones (labeled 0). Then, an in-house search algorithm was used to identify the interfacial computational nodes (voxels) delimiting the pore space, so that all the nodes in the multiphase system were classified in three groups: gas (0), water/gas and solid/gas interfacial nodes (1), and internal water and solid nodes (2). In the LBM simulations, diffusive dynamics were assigned to nodes labeled 0, a standard half-way bounce back scheme [68] to nodes labeled 1, and no dynamics to nodes labeled 2. In this way, solid and water phases were both treated as impermeable phases to gas transport.

According to Fick's law, the normalized effective diffusivity, $D_{\mathrm{j}}^{\text {eff }} / D_{\text {bulk }}$, was calculated as the ratio between the (constant) overall diffusive flux in the j-direction of interest and the overall bulk flux in that direction:

$$
\frac{D_{\mathrm{j}}^{\text {eff }}}{D_{\text {bulk }}}=\frac{\iint_{A} j_{\mathrm{j}} \mathrm{dA}}{A\left(D_{\text {bulk }} \Delta C_{\mathrm{j}} / L_{\mathrm{CD}, \mathrm{j}}\right)}
$$

where $A$ is the cross-sectional area of the examined GDL domain to the j-direction under study, and $j_{\mathrm{j}}, \Delta C_{\mathrm{j}}=1$, and $L_{\mathrm{CD}, \mathrm{j}}$ are the local diffusive flux in a computational node, the prescribed concentration difference, and the length of the computational domain (CD) in j-direction, respectively; note that $L_{\mathrm{CD}, \mathrm{j}}=L_{\mathrm{j}}+L_{\mathrm{in}}+L_{\mathrm{out}}$, with $L_{\mathrm{j}}$ the length of the GDL domain in j-direction, and $L_{\mathrm{in}} / L_{\text {out }}$ the lengths of the inlet/outlet gas zones padded to the GDL domain.

Simulations were started from a linear concentration profile and run until steady state, that is, when the relative variation of the volume-averaged concentration over a fixed 
time window of $10^{4}$ steps was below $10^{-6}$. This stop criterion assured negligible variations of the effective diffusivity. To exploit the parallel capabilities of the Palabos [66] implementation of LBM, computations were performed on the supercomputers Gullimin and Colosse; both managed by Calcul Québec and Compute Canada [71]. The number of processors was varied between 64 and 180, depending on the extent of the domain under study. Details on the validation campaign performed before the calculations on the GDL samples are provided as supplementary material. The computed concentration fields both in the in- and through-plane directions for the largest GDL wet sample $(1.3 \times 1.3 \times 0.28 \mathrm{~mm})$ are shown in Figure $6(\mathrm{~b})$ and $(\mathrm{c})$, respectively.

\subsection{Case Studies}

The segmented stacks were divided into numerous domains to study the impact of sample size and saturation distribution across the GDL. The domains explored in the simulation campaign are described below.

\subsubsection{Dry Conditions}

Four different cases were examined under dry conditions for both the untreated and PTFE-treated GDLs. Three domains including the full thickness of the segmented samples of $2 \times 2 \mathrm{~mm}\left(L_{z}=0.32 / 0.28 \mathrm{~mm}\right.$ for the $0 / 10 \%$ PTFE by weight GDLs), but different $x y$ sections were used to test the influence of sample size. These domains, as previously discussed in Section 3, do not incorporate the complete surface region of the GDL due to interferences of the membrane and the pipe with the irregular surface of the GDL. Additionally, an extra domain of $0.8 \times 0.8 \times 0.37 \mathrm{~mm}$, free of interfacial artifacts, was meticulously selected for each of the GDLs from the dry sample stacks. These additional GDL domains allowed us to explore the effect of the surface region excluded in the three previous cases. 


\subsubsection{Wet Conditions}

Under wet conditions, only the PTFE-treated GDL was investigated due to the better control of water in the invasion experiments. The same domains of $0.8 \times 0.8,1 \times 1$, and $1.3 \times 1.3 \times 0.28 \mathrm{~mm}$ used for dry diffusivity calculations were also examined under wet conditions. In addition, an extensive analysis was carried out on the smaller sample of $0.8 \times 0.8 \times 0.28 \mathrm{~mm}$ to investigate the impact of water distribution through the GDL on gas diffusive transport. As depicted in Figure 7, starting from the face next to the PTFE membrane, different cases were considered by gradually reducing the through-plane window length of the GDL included in the simulations, $\delta_{\mathrm{z}} \approx 0.056-0.28 \mathrm{~mm}$, with respect to the full thickness (FT) of the sample, $L_{\mathrm{z}}=0.28 \mathrm{~mm}$. In other words, a larger portion of the highly-saturated region near the injection pipe was progressively removed, so that the saturation variation in the numerical domain was decreased. The computed relative effective diffusivities, $g=D^{\text {eff,wet }} / D^{\text {eff,dry }}$, in all these thinner subdomains is aimed to provide fundamental information on the effect that the saturation distribution in finite-thickness GDLs has on diffusive transport. 


\section{Results and Discussion}

The following discussion of results is divided in two parts. First, in Section 5.1, the dry effective diffusivity of the untreated and PTFE-treated GDLs is examined. Then, the effective diffusivity of the PTFE-treated GDL under partially-saturated conditions is explored in Section 5.2. The diffusive properties in both the through- and in-plane directions of the material are investigated. Further details on the case studies can be consulted in Section 4.1.

\subsection{Dry Effective Diffusivity}

The computed results for the dry effective diffusivity in terms of the normalized function, $f=D^{\text {eff,dry }} / D_{\text {bulk }}$, along with the average porosity, $\varepsilon_{\text {avg }}$, of the analyzed GDL domains are listed in Table 1. Small changes in the dry effective diffusivity and porosity are observed for different domain sizes. The effective diffusivity is significantly higher in the in-plane direction due to the preferential alignment of fibers in the material plane. On average, the addition of PTFE decreases the effective diffusivity in the through-/in-plane directions $\left(f_{\mathrm{TP}} / f_{\mathrm{IP}}\right)$ from $0.25 / 0.41$ to $0.2 / 0.37$ due to the reduction of the porosity and the increase of the tortuosity of the material [8]. Besides, a notable increase of the effective diffusivity and the porosity is found in the samples fully including the core (CR) and the surface regions (SR) of the GDL $(0.31 / 0.54$ and $0.24 / 0.47$ for the untreated and PTFE-treated GDLs, respectively). The increase of the dry effective diffusivity in these domains can be explained by the lower transport resistance offered by the highly porous surface region as compared to the internal core region of the GDL [72]. In fact, this result suggests that the strong nonlinear effect of compression on the effective diffusivity of carbon-paper GDLs $[8,73]$ may be attributed to a large extent to the densification and depletion of the low-stiffness surface region $[72,74]$. The thin heterogeneous nature of GDL materials means that the surface region is a significant fraction of the material, which is an ever-present issue when dealing with finite-thickness porous media. 
The dry effective diffusivities obtained here are in good agreement with previous experimental data reported for Toray ${ }^{\circledR}$ carbon paper $[8-11,15]$. The computed effective diffusivities for the $\mathrm{CR}+\mathrm{SR}$ domains are similar to the values presented for uncompressed samples, whereas the computed effective diffusivities in the domains mostly excluding the surface region are in line with data reported for compressed GDLs of similar porosity. The effective diffusivity of Toray ${ }^{\circledR}$ TGP-H-120 over the porosity range analyzed here $\left(\varepsilon_{\mathrm{avg}}=0.66-0.75\right)$ can be well correlated by power laws of the form $f=\varepsilon_{\mathrm{avg}}^{\mathrm{n}}$, with $\mathrm{n}_{\mathrm{TP}} \approx 3.8$ and $\mathrm{n}_{\mathrm{IP}} \approx 2.3$ for the through- and in-plane directions, respectively [8-11,15]. The agreement between the present results and the available experimental data confirms the representativeness of the sample sizes used in the simulations, as well as the correct segmentation of the GDL samples. 


\subsection{Wet Effective Diffusivity}

\subsubsection{Through-Plane Direction}

The computed results for the partially-saturated GDL in the through-plane direction are presented in Figure $8(\mathrm{a})$-(b). The left panel shows the relative effective diffusivity, $g_{\mathrm{TP}}=$ $D_{\mathrm{TP}}^{\text {eff,wet }} / D_{\mathrm{TP}}^{\text {eff,dry }}$, as a function of average saturation, $s_{\mathrm{avg}}$, while the right panel shows the best-fit exponent $\mathrm{n}_{\mathrm{TP}}$ to a power-law of the form $g_{\mathrm{TP}}=\left(1-s_{\text {avg }}\right)^{\mathrm{n}_{\mathrm{TP}}}$, plotted as a function of the normalized through-plane window length, $\delta_{z} / L_{z}$, included in the numerical simulations (see sketch in Figure 7). The markers and colors between these two plots coincide, so it can be seen, for example, that the best-fit exponent $\mathrm{n}_{\mathrm{TP}}$ for the three widow lengths examined between $52 \%$ and $61 \%$ of the full thickness (FT) in Figure 8 (a) corresponds to $\mathrm{n}_{\mathrm{TP}} \approx 3$ in Figure $8(\mathrm{~b})$.

A striking feature of the results shown in Figure $8(a)-(b)$ is that the gas transport resistance of water at a given average saturation, as characterized by the exponent $\mathrm{n}_{\mathrm{TP}}$, never stabilizes as the peak saturation through the GDL decreases (the window length $\delta_{\mathrm{z}} / L_{\mathrm{z}}$ is reduced). This essentially shows that a unique power-law exponent $\mathrm{n}_{\mathrm{TP}}$ relating the relative effective diffusivity of partially-saturated GDLs with average saturation does not exist. The value of $\mathrm{n}_{\mathrm{TP}}$ fitted to the full-thickness domain data is about 7.5. Such strong resistance, even at low average saturations, is due to the severe bottleneck effect caused by the highly-flooded region close to the invasion face. It can be seen in Figure 8 (b) that this region of high diffusive resistance is extended up to about $5 \%$ of the full thickness with $\mathrm{n}_{\mathrm{TP}}>5$ (note that the high-resistance region would be somewhat thicker and $\mathrm{n}_{\mathrm{TP}}$ even higher if the complete surface region had been included in the segmented domain). In contrast, when the highly-saturated region near the invasion face is removed, the resistance of water to gas diffusive transport is sharply reduced. As shown in Figure $8(\mathrm{~b})$, the power-law exponent $\mathrm{n}_{\mathrm{TP}}$ in the GDL domains of low-to-middle diffusive resistance varies from about 4.5 to 1.5 , being the average value 3.1 . 
The inadequacy of the average saturation level to correlate the effective diffusivity fully can be more clearly illustrated qualitatively. Consider the schematic picture of throughplane saturation distributions in the GDL shown in Figure 9(a), corresponding to a linear and a flat saturation profile. In both cases, the average saturation is the same, $s_{\text {avg }}=$ $s_{\text {crit }} / 2$, where $s_{\text {crit }}$ is the local saturation level slightly below the gas percolation threshold. However, despite having the same average saturation, as shown in Figure 9(b), the effective diffusivity is dramatically reduced in the case of the linear profile due to the strong bottleneck effect caused by the high-saturation region. In contrast, for the flat profile there is no such bottleneck region. Introducing some numbers to this picture, if we assume that $s_{\text {crit }}=0.8\left(s_{\text {avg }}=0.4\right)$, and the relative effective diffusivities in the GDLs with the linear and flat saturation distributions are $g_{\mathrm{TP}}^{\text {lin }}=0.05$ and $g_{\mathrm{TP}}^{\text {flat }}=0.5$, the associated power-law exponents turn out to be $\mathrm{n}_{\mathrm{TP}}^{\text {lin }}=5.9$ and $\mathrm{n}_{\mathrm{TP}}^{\text {flat }}=1.4$, respectively. Similar differences can be observed in the numerical results. This simplistic but illustrative explanation shows that any measured effective diffusivity in partiallysaturated GDLs must explicitly consider the distribution of water in the sample, and not only the average amount. Such hypersensitivity to the saturation distribution stems from the finite-thickness nature of GDLs, spanning only 10-20 pores in the through-plane direction. The most basic tenets of percolation theory (infinite medium) and volume averaging (representative elementary volume) are not met in such thin materials [75]. Therefore, it should not be surprizing that the average saturation alone cannot fully characterize transport phenomena in finite-thickness GDLs.

To further illustrate the impact of the through-plane saturation distribution on the effective diffusivity, the exponent $\mathrm{n}_{T P}$ obtained by fitting each data point in Figure $8(\mathrm{a})$ is represented against different representative functions $\lambda(s)$ in Figure 10(a); $\lambda(s)=s_{\text {avg }}$ corresponds to the hollow blue circles. This plot clearly shows that the diffusive resistance caused by water is highly uncorrelated, e.g., for $\lambda(s)=s_{\text {avg }}=0.5$, where the exponent $\mathrm{n}_{\mathrm{TP}}$ ranges randomly from 2 to more than 7 . The variations of $\mathrm{n}_{\mathrm{TP}}$ can be better explained by introducing some metric that captures the basic features of the 
saturation distribution through the GDL, specifically the peak saturation. A normalized parameter $\lambda(s)=\Psi_{\mathrm{TP}}$ (solid green diamonds), can be defined as:

$$
\psi_{\mathrm{TP}}=\frac{\bar{s}_{Z}^{\max }-\bar{s}_{Z}^{\min }}{1-\bar{s}_{Z}^{\min }}
$$

where $\bar{s}_{Z}^{\min }$ and $\bar{s}_{Z}^{\max }$ are the minimum and maximum local saturation level across the GDL, respectively. The parameter $\psi_{\mathrm{TP}}$ measures the amplitude of the through-plane saturation distribution in the GDL, as compared to the maximum possible variation for a given value of $\bar{s}_{z}^{\min }$, that is, when $\bar{s}_{z}^{\max }=1$. Accordingly, $\Psi_{\mathrm{TP}}$ tends to 0 for a fully flat saturation profile $\left(\bar{s}_{Z}^{\max }=\bar{s}_{Z}^{\min }\right)$, while it approaches 1 when fully-saturated conditions are reached near the invasion face $\left(\bar{S}_{z}^{\max }=1\right)$. As can be seen in Figure 10(a), the exponents $n_{T P}$ collapse onto a single curve when they are expressed in terms of $\Psi_{T P}$ rather than $s_{\text {avg, }}$ and correlate quite solid over the whole range. The value of $\mathrm{n}_{\text {TP }}$ varies from about 1.5 when the saturation distribution in the GDL is nearly flat $\left(\psi_{\mathrm{TP}} \rightarrow 0\right)$, sharply increasing to more than 7 when a high-peak saturation distribution exists in the GDL $\left(\psi_{\mathrm{TP}} \rightarrow 1\right)$. These exponents are physically sound. They lie within the range of values for a porous medium composed of straight capillary tubes partially-filled with a water film on their walls $\left(\mathrm{n}_{\mathrm{TP}}=1\right)$, and a partially-saturated porous medium with no connected gas transport pathways $\left(\mathrm{n}_{\mathrm{TP}} \rightarrow \infty\right)$. This analysis further demonstrates that the inhomogeneous distribution of water and, in particular, the existence of local bottleneck regions in the GDL has major impacts on diffusive transport not taken into account just considering the average saturation.

The present data are compared to the available literature sources $[10,22,32]$ in Figure 11 , and a wide dispersion can be seen. The experimental data presented by Koresawa \& Utaka [32] are in the range of the results computed in the domains suffering from a severe bottleneck effect, which is not surprising given that they reported a high saturation region near their inlet using soft X-ray radiography [32]. In contrast, the works of Rosén et al. [22] and Hwang \& Weber [10] tend to agree more with the present results for the cases where the flooded inlet region was removed. The deviations of the 
results computed on the full-thickness domains with respect to both works $[10,22]$ could be anticipated. The numerical study by Rosén et al. [22] considered water distributions from an actual fuel cell. Hence, water did not invade into the full face of the GDL, unlike the present invasion experiments, which may resemble more the scenario found in a cell under severe flooding conditions (see discussion in Section 3.2.2). Moreover, Hwang \& Weber [10] controlled saturation by allowing water to evaporate from a fully-saturated GDL. Since GDLs are hydrophobic, evaporation of water corresponds to an imbibition of air, meaning that air enters the GDL via cracks and corners along the solid structure which are not filled by water. Therefore, it is likely that the water configurations of Hwang \& Weber [10] did not exhibit the large through-plane saturation variations observed in this work, which are characteristic of access-limited percolation processes. From the trends in Figure 11, it can be concluded that a power-law fit of the form $g_{\mathrm{TP}}=\left(1-s_{\mathrm{avg}}\right)^{\mathrm{n}_{\mathrm{TP}}}$ with and exponent $\mathrm{n}_{\mathrm{TP}} \approx 3$ falls through the middle of the data, which is in good agreement with the values presented in other numerical works $[7,21,23,33,36-39] ;$ all of them in the range from $\mathrm{n}_{\mathrm{TP}} \approx 2$ to $\mathrm{n}_{\mathrm{TP}} \approx 4$.

\subsubsection{In-Plane Direction}

The computed results in the in-plane direction for the same GDL domains analyzed in the previous section are shown in Figure 8(c)-(d). A much lower impact of the saturation distribution through the GDL on the in-plane relative effective diffusivity, $g_{\mathrm{IP}}=$ $D_{\mathrm{IP}}^{\text {eff,wet }} / D_{\mathrm{IP}}^{\text {eff,dry }}$, is observed. This different behavior arises from the perpendicular arrangement of transport processes in the material plane with respect to the throughplane saturation gradients. Hence, no important bottleneck effects are visible on inplane gas diffusion, and the power-law exponent $n_{\text {IP }}$ remains in all the cases between 2 and 3 .

Intriguingly, the results presented in Figure 8(d) show a progressive increase of the exponent $\mathrm{n}_{\mathrm{IP}}$ as the peak saturation across the $\mathrm{GDL}$ is decreased $\left(\delta_{\mathrm{z}} / L_{\mathrm{z}}\right.$ is reduced), opposite to the behavior shown in Figure $8(b)$ for $n_{T P}$. This effect can be qualitatively 
explained by the nonlinear effect of saturation on local transport processes, so that the diffusive flux in a region of low saturation is disproportionally higher than the diffusive flux in a region of either intermediate or high saturation. As illustrated in Figure 12, for a given average saturation, $s_{\text {avg }}$, the relative effective diffusivity, $g_{\mathrm{IP}}^{\mathrm{lin}}$, in a GDL with a linear saturation distribution is expected to be somewhat higher due to the high diffusive flux existing in the low-saturation region. In contrast, an intermediate diffusive resistance acts everywhere in a GDL with a flat saturation distribution, so that $g_{\mathrm{IP}}^{\text {flat }}$ is lower, i.e., the equivalent diffusive resistance is larger. Even though this phenomenon is less important than that observed for the through-plane direction, this effect again stresses that any transport property in finite-thickness porous media is highly influenced by the actual saturation distribution across the material and not only the average saturation level. A good correlation can be established between the exponent $\mathrm{n}_{\mathrm{IP}}$ and the parameter $\psi_{\mathrm{IP}}$, defined as:

$$
\psi_{\mathrm{IP}}=\frac{\operatorname{MAD}\left(\bar{s}_{\mathrm{z}}\right)}{S_{\mathrm{avg}}}
$$

where $\operatorname{MAD}\left(\bar{s}_{\mathrm{Z}}\right)$ is the mean absolute deviation of the saturation distribution across the GDL. The parameter $\psi_{\text {IP }}$ measures the variations of the local saturation level in the GDL from the average saturation, $s_{\mathrm{avg}}$, so that $\psi_{\mathrm{IP}}=0$ for a flat saturation profile. This definition is more suitable to characterize diffusive transport in the perpendicular direction to the saturation gradients unlike the one considered for the through-plane direction, which was based on the closeness of the maximum saturation, $\bar{s}_{Z}^{\max }$, towards 1 owing to the series arrangement of transport processes. Figure 10(b) shows the power-law exponent $n_{I P}$ as a function of the normalized parameter $\psi_{I P}^{*}=\psi_{I P} / \psi_{I P}^{\max }$, where $\psi_{I P}^{\max }$ is the maximum value in the dataset. As can be seen, the exponent $n_{I P}$ slightly increases as the saturation variation in the porous medium is lower $\left(\psi_{\mathrm{IP}}^{*}\right.$ decreases), thus in agreement with the qualitative explanation given before in Figure 12. The inadequacy of the average saturation, $s_{\text {avg }}$, to correlate the power-law exponent $n_{I P}$ is also clearly observed, although the spread of the data is lower compared to the through-plane direction due to the small changes of $n_{I P}$ in the numerical results. 
Figure 13 shows data previously presented in the literature for partially-saturated carbon-paper GDLs, along with the results computed in this work for the in-plane relative effective diffusivity. The data extracted from the literature correspond to the numerical studies by Zamel et al. [21], Rosén et al. [22], and Becker et al. [37]. No experimental works on the in-plane relative effective diffusivity of partially-saturated GDLs were found. Good agreement is observed between the present work and the results reported by other authors, with a much lower dispersion of the data than seen for the through-plane direction. Despite the slightly higher values of Becker et al. [37] and the lower values computed here for GDL domains with flatter saturation profiles (reduced window length $\delta_{z} / L_{z}$ ), the influence of saturation on in-plane diffusion is well characterized by a power-law of the form $g_{\mathrm{IP}}=\left(1-s_{\mathrm{avg}}\right)^{\mathrm{n}_{\mathrm{IP}}}$, with $\mathrm{n}_{\mathrm{IP}} \approx 2$. This value is also consistent with the pore network results of Gostick [33] and Nam \& Kaviany [7]. The good agreement of the data among all the authors further shows that the in-plane effective diffusivity is less influenced by the distribution of water across the GDL. Hence, a significant number of connected pathways for in-plane gaseous transport between and around water fingers should be expected for most operating conditions in fuel cells $[22,76]$. Note that this observation holds only for in-plane diffusion when water invades the GDL in the through-plane direction. This scenario is the most relevant for fuel-cell operation since water is generated at the catalyst layer and must percolate through the GDL to exit the cell. If water had been injected in the in-plane direction, however, one would expect similarly strong resistance to in-plane gas diffusion as in the results presented here for the through-plane direction. 


\section{Conclusions}

In this work, micrometer resolution X-ray computed tomography (XCT) was used to image dry and partially-saturated carbon-paper gas diffusion layers (Toray ${ }^{\circledR}$ TGP-H-120) at various levels of applied capillary pressure. The reconstructed image stacks were segmented into solid, air and liquid phases, and then used as computational domains for lattice Boltzmann (LB) simulations aimed to characterize the effective gas diffusivity. Excellent agreement was found between the computed dry effective diffusivity and previous experimental data in both the through- and in-plane directions. Under wet or partially-saturated conditions, diffusive transport in the GDL is strongly influenced by not only the amount of water but its distribution across the GDL. The dependence of the effective gas diffusivity with average saturation, $s_{\text {avg }}$, was well described by power laws of the form $\left(1-s_{\mathrm{avg}}\right)^{\mathrm{n}}$. However, it was observed that the exponent in the throughplane direction, $\mathrm{n}_{\mathrm{TP}}$, decreased systematically as the peak saturation in the GDL was lower. Extremely low effective diffusivities were found due to the bottleneck effect caused by the high-saturation region near the invasion face, leading to values of $\mathrm{n}_{\mathrm{TP}}>5$. When this region was removed, the power-law exponent $\mathrm{n}_{\mathrm{TP}}$ was strongly reduced, eventually reaching values around 2 for GDL domains with nearly flat saturation profiles. The average exponent $\mathrm{n}_{\mathrm{TP}}$ for saturation distributions with moderate peak values was about 3 in agreement with previous data reported in the literature. On the other hand, diffusive transport in the in-plane direction did not suffer from a bottleneck effect, leading to an exponent $\mathrm{n}_{\mathrm{IP}}$ about 2 but slightly increasing as the peak saturation was lower. This effect was explained by the nonlinear effect of water saturation on local transport processes, such that a sample with a flat saturation profile has a different equivalent resistance as the same sample with regions of high and low saturation, even if they have equal average saturation levels.

Two important consequences arise from the present findings. First, any determination of the effective diffusivity in finite-size GDLs should not only report the average saturation, 
but also an explicit description of the water configuration under which the measurements/calculations were made. This can take the form of ensuring well controlled water placement, or visualizations of the actual water distribution as was done here. Second, any realistic PEFC volume-averaged continuum model incorporating an ex-situ effective diffusivity vs. saturation relationship must be mindful of the particular conditions under which the correlation was determined to ensure its applicability to the modeled scenario.

In fact, the present findings suggest that volume-averaged multiphase models explicitly solving local diffusion processes within GDLs must incorporate constitutive relations obtained under conditions of no saturation gradient. This is because volume-averaged models predict their own saturation distributions, over which they evaluate the effective diffusivity at each computational node, implicitly assuming that all transport properties are locally homogeneous in every computational node. Hence, the application of any correlation determined globally in an inhomogeneous medium (i.e. with saturation gradients) to the virtually homogeneous computational nodes composing the effective medium is inconsistent. The development of a methodology for determining an 'intrinsic effective diffusivity' relationship suitable for volume-averaged continuum models remains an important challenge to be explored in future work. 


\section{Acknowledgements}

The authors thank Dr. Jin Hyun Nam (Daegu University, South Korea), Dr. Massoud Kaviany (University of Michigan, USA) and Dr. Felix N. Büchi (Paul Scherrer Institut, Switzerland) for insightful discussions and comments concerning their works $[7,22]$. The authors also thank Dr. Dula Parkinson and Dr. Alastair MacDowell at the Advanced Light Source (ALS) for help in obtaining and analyzing the tomographic images, as well as the support team of Calcul Québec and Compute Canada for their help during the simulation campaign. This work was supported by the Natural Science and Engineering Research Council of Canada's Discovery Grant program, the Assistant Secretary for Energy Efficiency and Renewable Energy, Fuel Cell Technologies Program, of the U. S. Department of Energy under contract DE-AC02-05CH11231, and Project ENE201124574 of the Spanish Ministerio de Economía y Competitividad (MEC). XCT experiments were performed in the beamline 8.3.2 at ALS, Lawrence Berkeley National Laboratory, which is a national user facility funded by the Department of Energy, Office of Basic Energy Sciences under contract DE-AC02-05CH11231. Numerical calculations were conducted in the supercomputing clusters Guillimin and Colosse managed by Calcul Québec and Compute Canada [71]. The operation of these supercomputers is funded by the Canada Foundation for Innovation (CFI), Ministère de l'Économie, de l'Innovation et des Exportations du Québec (MEIE), RMGA and the Fonds de recherche du Québec Nature et technologies (FRQ-NT). 


\section{Nomenclature}

\section{Symbols}

A GDL cross-sectional area [LB units]-[m $\left.{ }^{2}\right]$

$C \quad$ mass concentration [ $\left[\mathrm{B}\right.$ units] $\left[\mathrm{mol} / \mathrm{m}^{3}\right]$

c lattice velocity [LB units]-[m/s]

$c_{S} \quad$ lattice 'speed of sound' [LB units]-[m/s]

$D \quad$ mass diffusivity [ $L B$ units]-[ $\left[\mathrm{m}^{2} / \mathrm{s}\right]$

$d \quad$ diameter $[\mathrm{m}]$

$d_{\mathrm{p}} \quad$ pore diameter $[\mathrm{m}]$

$f \quad$ particle distribution function [LB units]

$f(\varepsilon) \quad$ normalized dry effective diffusivity function; see Eq. (3)

$g \quad$ gravitational acceleration $\left[\mathrm{m} / \mathrm{s}^{2}\right]$

$g(s) \quad$ relative effective diffusivity; see Eq. (3)

$h \quad$ height [m]

I grayscale intensity

$j \quad$ Fickian diffusion flux [LB units]-[mol$\left./ \mathrm{m}^{2} \mathrm{~s}\right]$

$L_{\mathrm{i}} \quad \mathrm{GDL}$ sample length in i-direction [LB units]-[m]

$\mathrm{n} \quad$ best-fit power-law exponent

$p \quad$ pressure $[\mathrm{Pa}]$

$p_{c} \quad$ capillary pressure $[\mathrm{Pa}]$

$R \quad$ radius $[\mathrm{m}]$

$s \quad$ liquid saturation

$\bar{s}_{x}\left(x_{\mathrm{i}}\right) \quad y z$-averaged liquid saturation at slice $x=x_{\mathrm{i}}$

$\bar{s}_{z}\left(z_{\mathrm{i}}\right) \quad x y$-averaged liquid saturation at slice $z=z_{\mathrm{i}}$

$t \quad$ time [LB units]-[s]

$\Delta t \quad$ time step [LB units]-[s]

$V \quad$ volume [LB units]-[ $\left[\mathrm{m}^{3}\right]$

$x \quad$ in-plane coordinate [LB units]-[m] 


$$
\begin{array}{ll}
\boldsymbol{x}_{\mathbf{i}} & \text { node position [LB units]-[m] } \\
\boldsymbol{\Delta} \boldsymbol{x} & \text { spatial increment [LB units]-[m] } \\
y & \text { secondary in-plane coordinate [LB units]-[m] } \\
z & \text { through-plane coordinate [LB units]-[m] }
\end{array}
$$

\section{Greek letters}

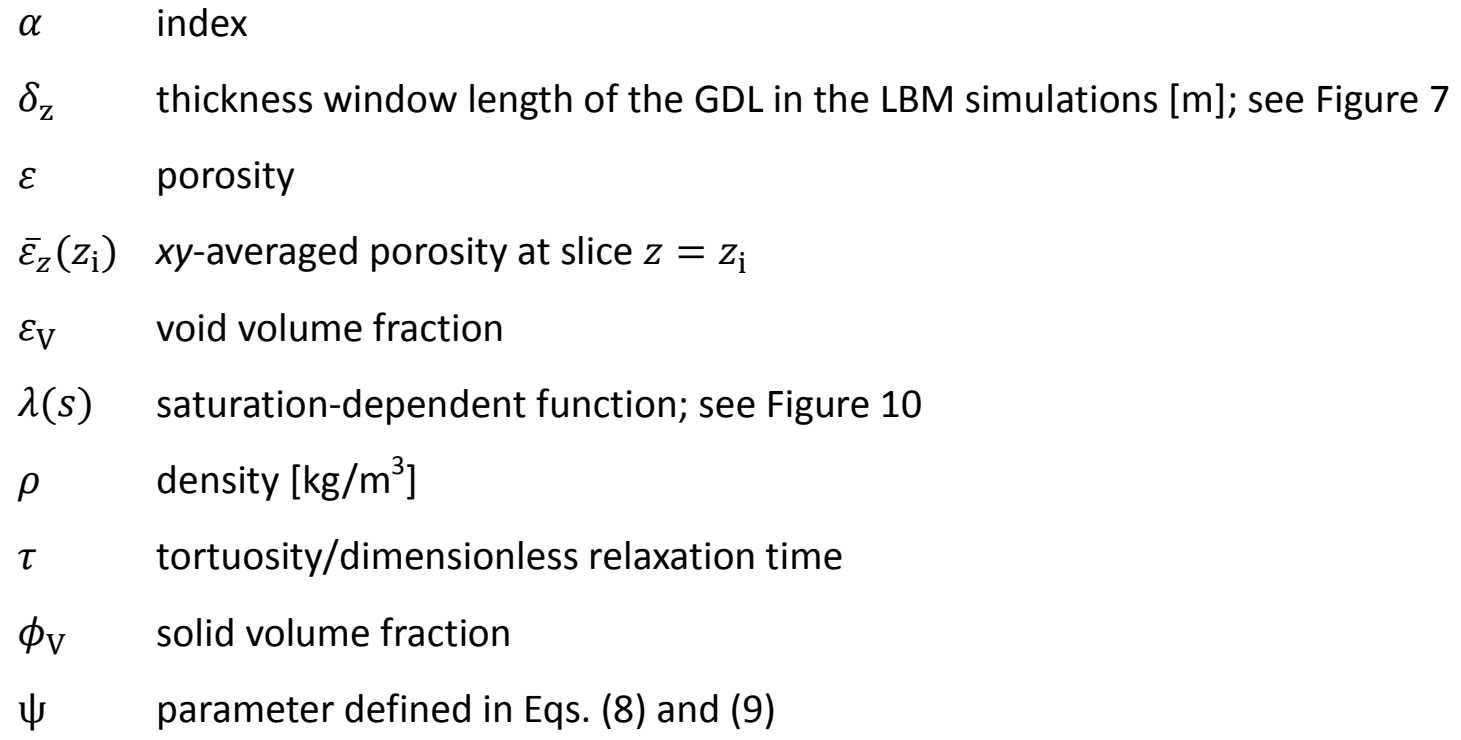

\section{Subscripts}

avg volume-averaged quantity in the whole sample

bulk bulk conditions

CD computational domain

crit critical local transport conditions; see Figures 9 and 12

$\mathrm{D} / \mathrm{C}$ dilation/contraction

F fiber

FT full thickness

g gas phase

IP in-plane direction

in inlet/inner

j direction of interest or under study 
I liquid phase

out outlet

$\mathrm{T}$ total

TP through-plane direction

th threshold

w water

\section{Superscripts}

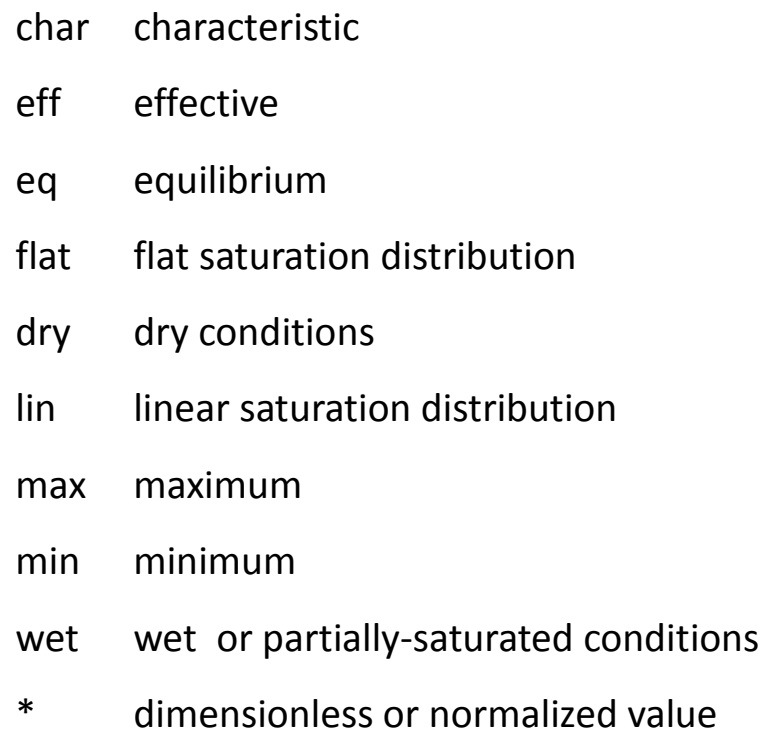




\section{References}

[1] M.W. Ellis, M.R. Von Spakovsky, D.J. Nelson, Fuel cell systems: efficient, flexible energy conversion for the 21st century, Proc. IEEE 89 (2001) 1808-1818.

[2] A. Casalegno, F. Bresciani, G. Groppi, R. Marchesi, Flooding of the diffusion layer in a polymer electrolyte fuel cell: Experimental and modelling analysis, J. Power Sources 196 (2011) 10632-10639.

[3] H. Li, Y. Tang, Z. Wang, Z. Shi, S. Wu, D. Song, J. Zhang, K. Fatih, J. Zhang, H. Wang, A review of water flooding issues in the proton exchange membrane fuel cell, J. Power Sources 178 (2008) 103-117.

[4] A.Z. Weber, J. Newman, Effects of Microporous Layers in Polymer Electrolyte Fuel Cells, J. Electrochem. Soc. 152 (2005) A677-A688.

[5] R. O'Hayre, S.-W. Cha, W. Colella, F.B. Prinz, Fuel Cell Fundamentals, John Wiley \& Sons, Inc., New York (2006).

[6] M. Kaviany, Principles of Heat Transfer in Porous Media, Springer, Berlin (1995).

[7] J.H. Nam, M. Kaviany, Effective diffusivity and water-saturation distribution in single- and two-layer PEMFC diffusion medium, Int. J. Heat Mass Transf. 46 (2003) 4595-4611.

[8] R. Flückiger, S.A. Freunberger, D. Kramer, A. Wokaun, G.G. Scherer, F.N. Büchi, Anisotropic, effective diffusivity of porous gas diffusion layer materials for PEFC, Electrochim. Acta 54 (2008) 551-559.

[9] J.M. LaManna, S.G. Kandlikar, Determination of effective water vapor diffusion coefficient in pemfc gas diffusion layers, Int. J. Hydrogen Energy 36 (2011) 50215029.

[10] G.S. Hwang, A.Z. Weber, Effective-Diffusivity Measurement of Partially-Saturated Fuel-Cell Gas-Diffusion Layers, J. Electrochem. Soc. 159 (2012) F683-F692.

[11] M.J. Martínez, S. Shimpalee, J.W. Van Zee, Measurement of MacMullin Numbers for PEMFC Gas-Diffusion Media, J. Electrochem. Soc. 156 (2009) B80-B85.

[12] M.J. Martínez-Rodríguez, T. Cui, S. Shimpalee, S. Seraphin, B. Duong, J.W. Van Zee, Effect of microporous layer on MacMullin number of carbon paper gas diffusion layer, J. Power Sources 207 (2012) 91-100. 
[13] M. Möst, M. Rzepka, U. Stimming, Analysis of the diffusive mass transport in the anode side porous backing layer of a direct methanol fuel cell, J. Power Sources 191 (2009) 456-464.

[14] G. Unsworth, L. Dong, X. Li, Improved experimental method for measuring gas diffusivity through thin porous media, AIChE J. 59 (2013) 1409-1419.

[15] N. Zamel, N.G.C. Astrath, X. Li, J. Shen, J. Zhou, F.B.G. Astrath, H. Wang, Z.-S. Liu Experimental measurements of effective diffusion coefficient of oxygen-nitrogen mixture in PEM fuel cell diffusion media, Chem. Eng. Sci. 65 (2010) 931-937.

[16] C. Chan, N. Zamel, X. Li, J. Shen, Experimental measurement of effective diffusion coefficient of gas diffusion layer/microporous layer in PEM fuel cells, Electrochim. Acta 65 (2012) 13-21.

[17] Y. Utaka, Y. Tasaki, S. Wang, T. Ishiji, S. Uchikoshi, Method of measuring oxygen diffusivity in microporous media, Int. J. Heat Mass Transf. 52 (2009) 3685-3692.

[18] D. Kramer, S.A. Freunberger, R. Flückiger, I.A. Schneider, A. Wokaun, F.N. Büchi, G.G. Scherer, Electrochemical diffusimetry of fuel cell gas diffusion layers, J. Electroanal. Chem. 612 (2008) 63-77.

[19] R. Rashapov, F. Imami, J.T. Gostick, A method for measuring in-plane effective diffusivity in thin porous media, Int. J. Heat Mass Transf. 85 (2015) 367-374.

[20] N. Zamel, X. Li, J. Shen, Correlation for the Effective Gas Diffusion Coefficient in Carbon Paper Diffusion Media, Energy \& Fuels 23 (2009) 6070-6078.

[21] N. Zamel, X. Li, J. Becker, A. Wiegmann, Effect of liquid water on transport properties of the gas diffusion layer of polymer electrolyte membrane fuel cells, Int. J. Hydrogen Energy 36 (2011) 5466-5478.

[22] T. Rosén, J. Eller, J. Kang, N.I. Prasianakis, J. Mantzaras, F.N. Büchi, Saturation Dependent Effective Transport Properties of PEFC Gas Diffusion Layers, J. Electrochem. Soc. 159 (2012) F536-F544.

[23] R. Wu, Q. Liao, X. Zhu, H. Wang, A fractal model for determining oxygen effective diffusivity of gas diffusion layer under the dry and wet conditions, Int. J. Heat Mass Transf. 54 (2011) 4341-4348.

[24] Y. Shi, J. Xiao, S. Quan, M. Pan, L. Zhang, Fractal model for prediction of effective hydrogen diffusivity of gas diffusion layer in proton exchange membrane fuel cell, Int. J. Hydrogen Energy 35 (2010) 2863-2867. 
[25] Y. Shi, H. Wu, S. Quan, J. Xiao, M. Pan, Fractal model for predicting the effective binary oxygen diffusivity of the gas diffusion layer in proton exchange membrane fuel cells, J. Power Sources 195 (2010) 4865-4870.

[26] D. Shou, J. Fan, F. Ding, Effective diffusivity of gas diffusion layer in proton exchange membrane fuel cells, J. Power Sources 225 (2013) 179-186.

[27] D. Shou, J. Fan, M. Mei, F. Ding, An analytical model for gas diffusion though nanoscale and microscale fibrous media, Microfluidics Nanofluidics 16 (2013) 381-389.

[28] P.K. Das, X. Li, Z.-S. Liu, Effective transport coefficients in PEM fuel cell catalyst and gas diffusion layers: Beyond Bruggeman approximation, Appl. Energy 87 (2010) 2785-2796.

[29] D.A.G. Bruggeman, Berechnung verschiedener physikalischer Konstanten von heterogenen Substanzen. I. Dielektrizitätskonstanten und Leitfähigkeiten der Mischkörper aus isotropen Substanzen, Annalen der Physik 5 (1935) 636-664.

[30] M.M. Tomadakis, S.V. Sotirchos, Ordinary and transition regime diffusion in random fiber structures, AIChE J. 39 (1993) 397-412.

[31] Y. Utaka, I. Hirose, Y. Tasaki, Characteristics of oxygen diffusivity and water distribution by $\mathrm{X}$-ray radiography in microporous media in alternate porous layers of different wettability for moisture control in gas diffusion layer of PEFC, Int. J. Hydrogen Energy 36 (2011) 9128-9138.

[32] R. Koresawa, Y. Utaka, Precise measurement of effective oxygen diffusivity for microporous media containing moisture by review of galvanic cell oxygen absorber configuration, Int. J. Heat Mass Transf. 76 (2014) 549-558.

[33] J.T. Gostick, Random Pore Network Modeling of Fibrous PEMFC Gas Diffusion Media Using Voronoi and Delaunay Tessellations, J. Electrochem. Soc. 160 (2013) F731-F743.

[34] J.T. Gostick, M.A. Ioannidis, M.W. Fowler, M.D. Pritzker, Pore network modeling of fibrous gas diffusion layers for polymer electrolyte membrane fuel cells, J. Power Sources 173 (2007) 277-290.

[35] R. Wu, Q. Liao, X. Zhu, H. Wang, Y.-d. Ding, J. Li, D.-d. Ye, A Two-dimensional Network Study on Oxygen Transport in Porous Gas Diffusion Layer, Fuel Cells 11 (2011) 255-262. 
[36] R. Wu, X. Zhu, Q. Liao, H. Wang, Y. Ding, J. Li, D.-d. Ye, Determination of oxygen effective diffusivity in porous gas diffusion layer using a three-dimensional pore network model, Electrochim. Acta 55 (2010) 7394-7403.

[37] J. Becker, V. Schulz, A. Wiegmann, Numerical Determination of Two-Phase Material Parameters of a Gas Diffusion Layer Using Tomography Images, J. Fuel Cell Sci. Technol. 5 (2008) 021006 1-9.

[38] A. Iranzo, P. Boillat, P. Oberholzer, J. Guerra, A novel approach coupling neutron imaging and numerical modelling for the analysis of the impact of water on fuel cell performance, Energy 68 (2014) 971-981.

[39] J.P. Owejan, T.A. Trabold, M.M. Mench, Oxygen transport resistance correlated to liquid water saturation in the gas diffusion layer of PEM fuel cells, Int. J. Heat Mass Transf. 71 (2014) 585-592.

[40] C.-Y. Wang, Fundamental models for fuel cell engineering., Chem. Rev. 104 (2004) 4727-65.

[41] A.Z. Weber, R.L. Borup, R.M. Darling, P.K. Das, T.J. Dursch, W. Gu, D. Harvey, A. Kusoglu, S. Litster, M.M. Mench, R. Mukundan, J.P. Owejan, J.G. Pharoah, M. Secanell, I.V. Zenyuk, A Critical Review of Modeling Transport Phenomena in Polymer-Electrolyte Fuel Cells, J. Electrochem. Soc. 161 (2014) F1254-F1299.

[42] P.A. García-Salaberri, M. Vera, I. Iglesias, Modeling of the anode of a liquid-feed DMFC: Inhomogeneous compression effects and two-phase transport phenomena, J. Power Sources 246 (2014) 239-252.

[43] S. Succi, E. Foti, F. Higuera, Three-dimensional flows in complex geometries with the lattice Boltzmann method, Europhys. Lett. 10 (1989) 433-438.

[44] J. Park, M. Matsubara, X. Li, Application of lattice Boltzmann method to a microscale flow simulation in the porous electrode of a PEM fuel cell, J. Power Sources 173 (2007) 404-414.

[45] T. Koido, T. Furusawa, K. Moriyama, An approach to modeling two-phase transport in the gas diffusion layer of a proton exchange membrane fuel cell, J. Power Sources 175 (2008) 127-136.

[46] G. Inoue, T. Yoshimoto, Y. Matsukuma, M. Minemoto, Development of simulated gas diffusion layer of polymer electrolyte fuel cells and evaluation of its structure, J. Power Sources 175 (2008) 145-158. 
[47] L. Hao, P. Cheng, Lattice Boltzmann simulations of anisotropic permeabilities in carbon paper gas diffusion layers, J. Power Sources 186 (2009) 104-114.

[48] P. Rama, Y. Liu, R. Chen, H. Ostadi, K. Jiang, X. Zhang, Y. Gao, Determination of the anisotropic permeability of a carbon cloth gas diffusion layer through X-ray computer micro-tomography and single-phase lattice Boltzmann, Int. J. Numer. Meth. Fluids 67 (2011) 518-530.

[49] J. Yablecki, A. Nabovati, A. Bazylak, Modeling the Effective Thermal Conductivity of an Anisotropic Gas Diffusion Layer in a Polymer Electrolyte Membrane Fuel Cell, J. Electrochem. Soc. 159 (2012) B647-B653.

[50] A. Groso, R. Abela, M. Stampanoni, Implementation of a fast method for high resolution phase contrast tomography, Opt. Express 14 (2006) 8103-8110.

[51] A.D. Santamaria, P.K. Das, J.C. MacDonald, A.Z. Weber, Liquid-Water Interactions with Gas-Diffusion-Layer Surfaces, J. Electrochem. Soc. 161 (2014) F1184-F1193.

[52] J.T. Gostick, M.A. Ioannidis, M.W. Fowler, M.D. Pritzker, Wettability and capillary behavior of fibrous gas diffusion media for polymer electrolyte membrane fuel cells, J. Power Sources. 194 (2009) 433-444.

[53] D. Sage, D. Prodanov, J.-Y. Tinevez, J. Schindelin, ImageJ User \& Developer Conference (2012), Luxembourg.

[54] Toray carbon fiber paper "TGP-H" Datasheet, Toray Industries, Inc, Advanced Composites Dept., Tokyo (2001).

[55] N. Otsu, A Threshold Selection Method from Gray-Level Histograms, IEEE Trans. on systems, man, and cybernetics 9 (1979) 62-66.

[56] S. Paris, F. Durand, A Fast Approximation of the Bilateral Filter Using a Signal Processing Approach, Int. J. Comput. Vis. 81 (2009) 24-52.

[57] V. Radhakrishnan, P. Haridoss, Effect of cyclic compression on structure and properties of a Gas Diffusion Layer used in PEM fuel cells, Int. J. Hydrogen Energy 35 (2010) 11107-11118.

[58] A. El-kharouf, T.J. Mason, D.J.L. Brett, B.G. Pollet, Ex-situ characterisation of gas diffusion layers for proton exchange membrane fuel cells, J. Power Sources 218 (2012) 393-404.

[59] Z. Fishman, A. Bazylak, Heterogeneous Through-Plane Porosity Distributions for Treated PEMFC GDLs I. PTFE Effect, J. Electrochem. Soc. 158 (2011) B841-B845. 
[60] Z. Fishman, J. Hinebaugh, a. Bazylak, Microscale Tomography Investigations of Heterogeneous Porosity Distributions of PEMFC GDLs, J. Electrochem. Soc. 157 (2010) B1643-B1650.

[61] R. Flückiger, F. Marone, M. Stampanoni, A. Wokaun, F.N. Büchi, Investigation of liquid water in gas diffusion layers of polymer electrolyte fuel cells using X-ray tomographic microscopy, Electrochim. Acta 56 (2011) 2254-2262.

[62] J.T. Gostick, M.W. Fowler, M.D. Pritzker, M.A. Ioannidis, L.M. Behra, In-plane and through-plane gas permeability of carbon fiber electrode backing layers, J. Power Sources 162 (2006) 228-238.

[63] J. Hinebaugh, Z. Fishman, A. Bazylak, Unstructured Pore Network Modeling with Heterogeneous PEMFC GDL Porosity Distributions, J. Electrochem. Soc. 157 (2010) B1651-B1657.

[64] S. Succi, The Lattice-Boltzmann Equation for Fluid Dynamics and Beyond, Oxford University Press, New York (2001).

[65] F. Higuera, J. Jimenez, Boltzmann approach to lattice gas simulations, Europhys. Lett. 9 (1989) 663-668.

[66] Palabos 1.3r0, FlowKit Ltd. (http://www.palabos.org/).

[67] P.L. Bhatnagar, E.P. Gross, M. Krook, A Model for Collision Processes in Gases. I. Small Amplitude Processes in Charged and Neutral One-Component Systems, Phys. Rev. 94 (1954) 511-525.

[68] D.A. Wolf-Gladrow, Lattice Gas Cellular Automata and Lattice Boltzmann Models, Springer, Berlin (2000).

[69] Z. Guo, B. Shi, C. Zheng, A coupled lattice BGK model for the Boussinesq equations, Int. J. Numer. Meth. Fluids 39 (2002) 325-342.

[70] Q. Zou, X. He, On pressure and velocity boundary conditions for the lattice Boltzmann BGK model, Phys. Fluids 9 (1997) 1591-1598.

[71] http://calculquebec.ca

[72] Z. Fishman, A. Bazylak, Heterogeneous Through-Plane Distributions of Tortuosity, Effective Diffusivity, and Permeability for PEMFC GDLs, J. Electrochem. Soc. 158 (2011) B247-B252. 
[73] P.A. García-Salaberri, M. Vera, R. Zaera, Nonlinear orthotropic model of the inhomogeneous assembly compression of PEM fuel cell gas diffusion layers, Int. J. Hydrogen Energy 36 (2011) 11856-11870.

[74] P.R. Challa, X-ray Investigations of PEMFC Gas Diffusion Layers (GDLs), Master's Thesis, University of Toronto (2012).

[75] M. Rebai, M. Prat, Scale effect and two-phase flow in a thin hydrophobic porous layer. Application to water transport in gas diffusion layers of proton exchange membrane fuel cells, J. Power Sources 192 (2009) 534-543.

[76] J.P. Owejan, T.A. Trabold, D.L. Jacobson, D.R. Baker, D.S. Hussey, M. Arif, In situ investigation of water transport in an operating PEM fuel cell using neutron radiography: Part 2 - Transient water accumulation in an interdigitated cathode flow field, Int. J. Heat Mass Transf. 49 (2006) 4721-4731. 


\section{List of Tables and Figures}

Table 1: Computed through-plane (TP) and in-plane (IP) normalized dry effective diffusivities, $f=D^{\text {eff,dry }} / D_{\text {bulk }}$, for the untreated and 10\% PTFE-treated GDLs corresponding to domains with different $x y$-sections but mostly excluding the surface region of the material, and comparison with domains including both the core and the surface region (CR+SR) of the GDLs; see Figure $3(b)$ for details. The average porosity, $\varepsilon_{\text {avg }}$, of each GDL domain, and the average exponents $\mathbf{n}_{\mathrm{TP}} / \mathbf{n}_{\text {IP }}$ from a fit of the numerical results to a power law of the form $\boldsymbol{f}=\varepsilon_{\text {avg }}^{\mathrm{n}}$ are also included.

Figure 1: (a) Schematic of the capillary pressure cell used in the water-invasion experiments, illustrating sample position and X-ray path, and (b) micron resolution reconstructed crosssection of the GDL, showing the direction of the $x-z$ coordinate axis. The pipes above and below the GDL are visible, as is the PTFE membrane on the sample.

Figure 2: Cross-sectional view of the $2 \times 2 \times 0.28 \mathrm{~mm}$ segmented sample of $10 \%$ PTFE-treated carbon paper (capillary pressure $p_{c}=4 \mathrm{kPa}$ ) at different steps of the segmentation process: (a) original grayscale image; (b) a base segmentation of the solid phase is extracted from the dry sample stack applying Otsu's method [55]; (c) the imperfect matching of the solid structure is corrected using a 3D dilation/contraction morphological operation; (d) darkened regions in water fingers are removed applying a 3D bilateral filter; (e) water is differentiated by manual thresholding, and the final segmented stack is obtained by combining the results of the solid phase and water (see supplementary material for further details). 
Figure 3: (a) Average mercury saturation, $s_{\text {avg }}$, as a function of pore diameter, $d_{\mathrm{p}}$, computed by the MIO algorithm of Gostick [33] on the untreated and 10\% PTFE-treated segmented dry samples of $2 \times 2 \times 0.32$ and $2 \times 2 \times 0.28 \mathrm{~mm}$, as compared to previous experimental data $[14,57,58]$. The characteristic pore diameter, $d_{\mathrm{p}}^{\text {char }}$, of Toray ${ }^{\circledR}$ TGP-H-120 is between 20-35 $\mu \mathrm{m}$. (b) $x y$-averaged local porosity distribution, $\bar{\varepsilon}_{\mathrm{z}}$, across the GDL (z-direction) corresponding to the complete set of domains used for dry diffusivity calculations. The case studies are composed of three domains with different $x y$-sections $(0.8 \times 0.8,1 \times 1$ and $1.3 \times 1.3 \mathrm{~mm})$ extracted from the large segmented samples of $2 \times 2 \mathrm{~mm}$ mostly excluding the surface region (SR) of the GDL, and two additional domains of $0.8 \times 0.8 \times 0.37 \mathrm{~mm}$ carefully selected from the dry sample stacks to include both the core and surface regions (CR+SR).

Figure 4: Variation of the average water saturation level, $s_{\mathrm{avg}}$, with capillary pressure, $\boldsymbol{p}_{\mathrm{c}}$, in the present water-invasion experiments for the $10 \%$ PTFE-treated GDL, as compared to the experimental data of Gostick et al. [52]. The average saturation level was calculated on the large segmented sample of $2 \times 2 \times 0.28 \mathrm{~mm}$. The $+/-0.5 \mathrm{kPa}$ error bars accounts for the resolution of the ruler $(+/-0.5 \mathrm{~mm})$ used to measure the static head of water in the invasion experiments.

Figure 5: Local distributions of water saturation along (a) the through-plane direction $\left(\overline{\mathbf{s}}_{\mathbf{z}}\right.$, locally averaged in each $x y$-slice), and (b) the in-plane direction $\left(\bar{s}_{\mathbf{x}}\right.$, locally averaged in each $y z$ slice) at different applied capillary pressures, $p_{c}$, for a $10 \%$ PTFE-treated GDL domain of $0.8 \times 0.8 \times 0.28 \mathrm{~mm}$ centered on the injection pipe. The average saturation level, $s_{\text {avg, }}$ in the whole domain is indicated in brackets. The breakthrough point in the invasion experiments was reached around $p_{\mathrm{c}} \approx 3-4 \mathrm{kPa}$.

Figure 6: (a) Computational domain used for effective gas diffusivity calculations with the LBM, showing the different elements of the system. (b) and (c) Computed concentration fields corresponding to simulations in the in- and through-plane directions, respectively. The resolution of the $1.3 \times 1.3 \times 0.28 \mathrm{~mm} 10 \%$ PTFE-treated GDL domain was downscaled by a factor $3 \times 3 \times 1$ to avoid memory limitations of the $48 \mathrm{~GB}$ rendering workstation. 
Figure 7: Sketch of the study performed to examine the impact of the saturation distribution across the GDL, $\bar{s}_{\mathrm{z}}$, on the relative effective diffusivity, $g=D^{\text {eff,wet }} / D^{\text {eff,dry }}$, in a $0.8 \times 0.8 \times 0.28$ $\mathrm{mm} \mathrm{10 \%} \mathrm{PTFE-treated} \mathrm{domain} \mathrm{centered} \mathrm{on} \mathrm{the} \mathrm{injection} \mathrm{pipe.} \mathrm{Starting} \mathrm{from} \mathrm{the} \mathrm{face} \mathrm{adjacent}$ to the PTFE membrane, multiple thinner subdomains are examined by varying the throughplane window length of the GDL, $\delta_{\mathrm{z}}$, with respect to the full thickness (FT). That is, larger portions of the GDL near the invasion face are gradually removed. The extent of the thinner subdomains in the $x y$-plane remains unaltered. See Figure $\mathbf{5}$ for the saturation profiles in the entire range of applied capillary pressures.

Figure 8: Left panel: computed relative effective diffusivity, $g=D^{\text {eff,wet }} / D^{\text {eff,dry }}$, in (a) the through-plane (TP), and (c) the in-plane (IP) directions, as a function of average saturation, $s_{\text {avg, }}$ corresponding to the full-thickness (FT) $10 \%$ PTFE-treated domains with different $x y$ sections $(1.3 \times 1.3,1 \times 1,0.8 \times 0.8 \times 0.28 \mathrm{~mm})$, and thinner subdomains with different throughplane window lengths, $\delta_{\mathrm{z}} / L_{\mathrm{z}}$, belonging to the $0.8 \times 0.8 \times 0.28 \mathrm{~mm} \mathrm{GDL}$ domain (colored markers); see Section 4.1 for details. Right panel: variation of the best-fit exponent $\mathbf{n}$ to a power-law of the form $g=\left(1-s_{\mathrm{avg}}\right)^{\mathrm{n}}$, as a function of the normalized window length, $\delta_{\mathrm{z}} / L_{\mathrm{z}}$, for the computed results in (b) the through-plane, and (d) the in-plane directions on the $0.8 \times 0.8 \times 0.28 \mathrm{~mm}$ domain.

Figure 9: Schematic of the effect of water distribution across the GDL on through-plane (TP) diffusive transport: (a) qualitative differences between two GDLs partially-saturated with the same average saturation, $s_{\text {avg }}=s_{\text {crit }} / 2$, but with a linear and a flat saturation profile, $\bar{s}_{\mathrm{z}}$, across the porous medium (z-direction), and (b) consequences of the above differences on the through-plane relative effective diffusivity, $g_{\mathrm{TP}}=D_{\mathrm{TP}}^{\text {eff,wet }} / D_{\mathrm{TP}}^{\text {eff,dry }} ; s_{\text {crit }}$ is the local saturation level slightly below the gas percolation threshold. The high-saturation region strongly limiting the through-plane gas diffusive flux in the linear profile is indicated in orange. 
Figure 10: Variation of the power-law exponent, $n=\log (g) / \log \left(1-s_{\mathrm{avg}}\right)$, associated to each data point $\left(s_{\mathrm{avg}}, g\right)$ presented in Figure 8(a) and (c) with two different saturation-dependent functions $\lambda(s)$ for (a) the through-plane (TP), and (b) the in-plane (IP) directions: $\lambda(s)=s_{\text {avg }}$ (average saturation) corresponds to the hollow blue circles, and $\lambda(s)=\psi$ to the solid green diamonds (see Eqs. (8) and (9)); $\psi_{\mathrm{IP}}^{\max }$ is the maximum value in the dataset. Only the results computed at capillary pressures above the breakthrough point are included ( $p_{c} \geq 3 \mathbf{k P a}$ ).

Figure 11: Through-plane (TP) relative effective diffusivity, $g_{\mathrm{TP}}=D_{\mathrm{TP}}^{\text {eff,wet }} / D_{\mathrm{TP}}^{\text {eff,dry }}$, as a function of average saturation, $s_{\text {avg }}$, reported in previous works for carbon-paper GDLs $[10,22,32]$, as compared to the present numerical results. The data computed on the GDL domains including the flooded inlet region of high diffusive resistance (solid black diamonds) are differentiated from the remaining results (hollow black diamonds). See Figure 8(a)-(b) for further details.

Figure 12: Schematic of the effect of water distribution across the GDL on the in-plane (IP) relative effective diffusivity, $g_{\mathrm{IP}}=D_{\mathrm{IP}}^{\text {eff,wet }} / D_{\mathrm{IP}}^{\text {eff,dry }}$. See caption to Figure 9 for details. The yellow/orange color map indicating the local resistance to in-plane gas diffusion is non-linearly scaled according to the expression: $\left[\left(s_{\text {crit }}-\bar{s}_{\mathbf{z}}\right) / s_{\text {crit }}\right]^{2}$.

Figure 13: In-plane (IP) relative effective diffusivity, $g_{\mathrm{IP}}=D_{\mathrm{IP}}^{\text {eff,wet }} / D_{\mathrm{IP}}^{\text {eff,dry }}$, as a function of average saturation, $\boldsymbol{s}_{\mathrm{avg}}$, reported in previous studies for carbon-paper GDLs [21,22,37], as compared to the present numerical results (hollow black diamonds). See Figure 8 (c)-(d) for further details. 
Domains mostly excluding SR (different $x y$-section)

\begin{tabular}{|c|c|c|c|c|c|}
\hline & \multicolumn{3}{|c|}{$f_{\mathrm{TP}} / f_{\mathrm{IP}}\left(\varepsilon_{\mathrm{avg}}\right)$} & $f_{\mathrm{TP}} / f_{\mathrm{IP}}\left(\varepsilon_{\mathrm{avg}}\right)$ & \\
\hline PTFE & $0.8 \times 0.8 \mathrm{~mm}$ & $1.0 \times 1.0 \mathrm{~mm}$ & $1.3 \times 1.3 \mathrm{~mm}$ & $0.8 \times 0.8 \mathrm{~mm}$ & $\mathbf{n}_{\mathrm{TP}} / \mathbf{n}_{\mathrm{IP}}$ (average) \\
\hline $0 \%$ & $0.26 / 0.42(0.69)$ & 0.24 / $0.41(0.69)$ & $0.25 / 0.40(0.69)$ & $0.31 / 0.54(0.75)$ & \multirow{2}{*}{$3.85 / 2.32$} \\
\hline $10 \%$ & $0.20 / 0.37(0.66)$ & $0.20 / 0.38(0.66)$ & $0.21 / 0.37(0.66)$ & $0.24 / 0.47(0.70)$ & \\
\hline
\end{tabular}



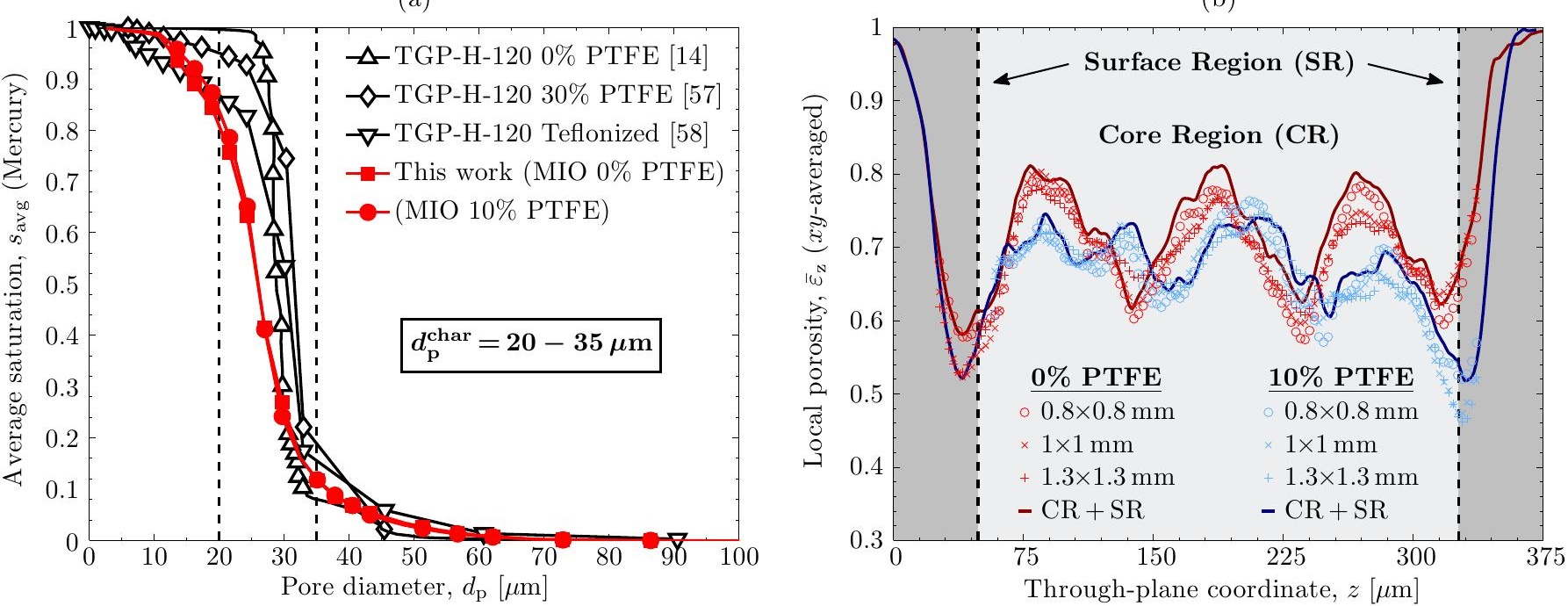


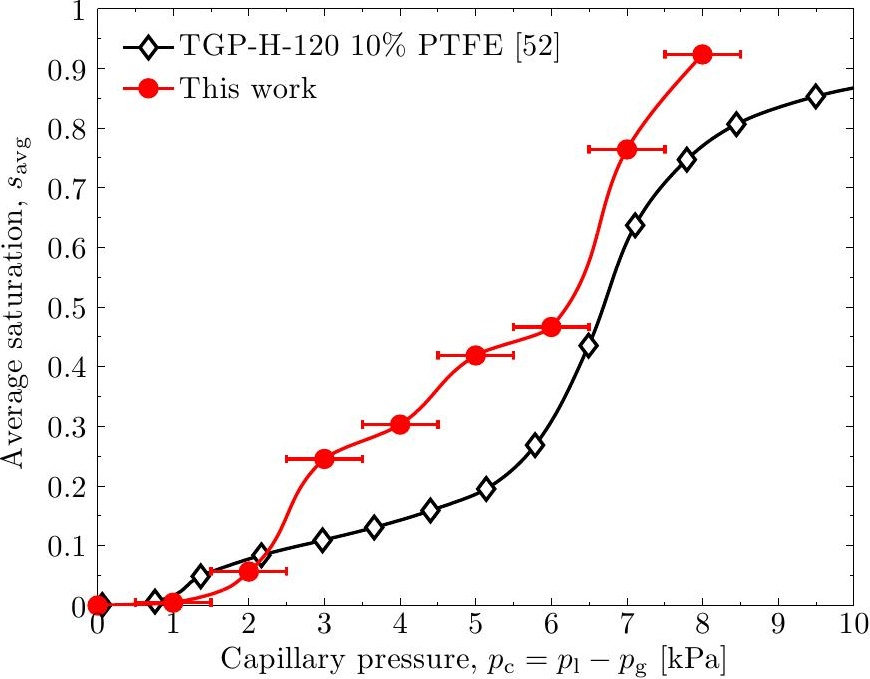



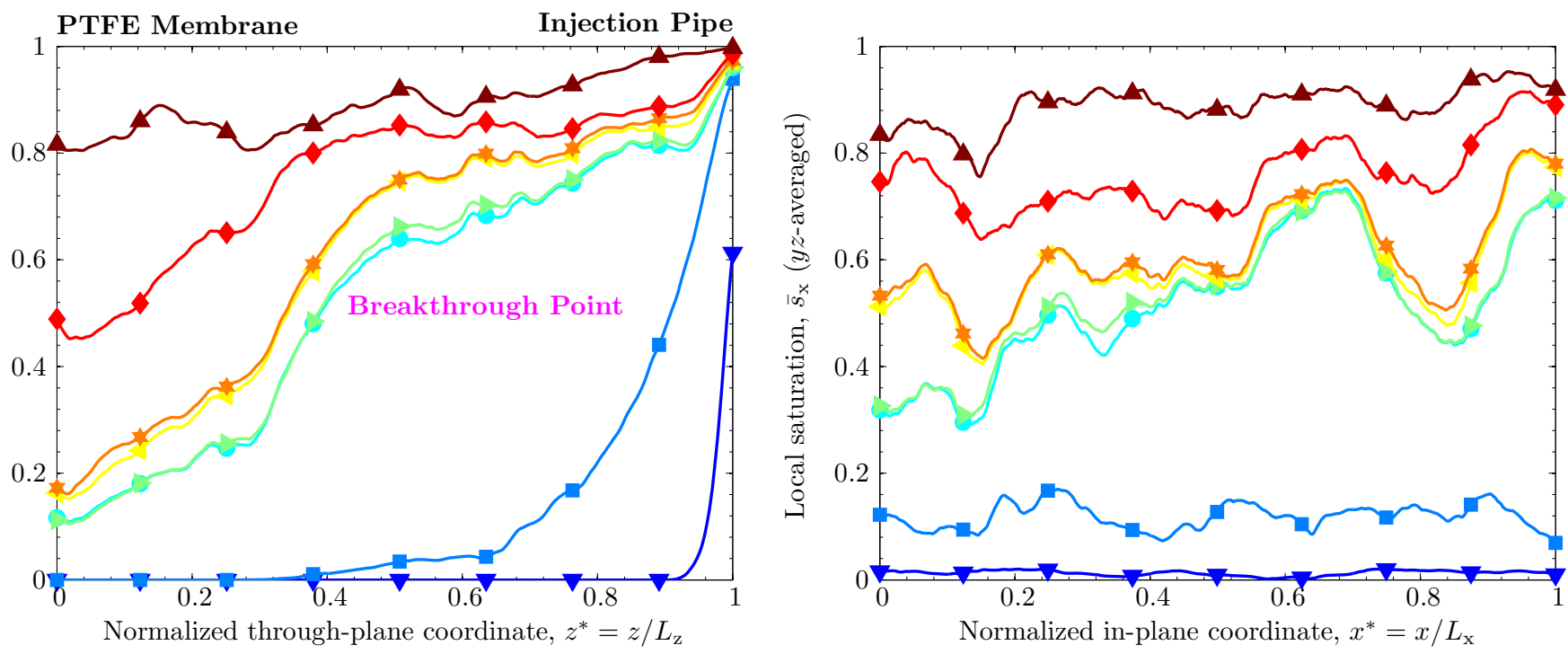

$\boldsymbol{\nabla} p_{\mathrm{c}}=1 \mathrm{kPa}\left(s_{\mathrm{avg}}=0.01\right)-2(0.12)-{ }^{-}-3(0.52)$

$-4(0.53)$

$5(0.61) \div-6(0.62)$ 


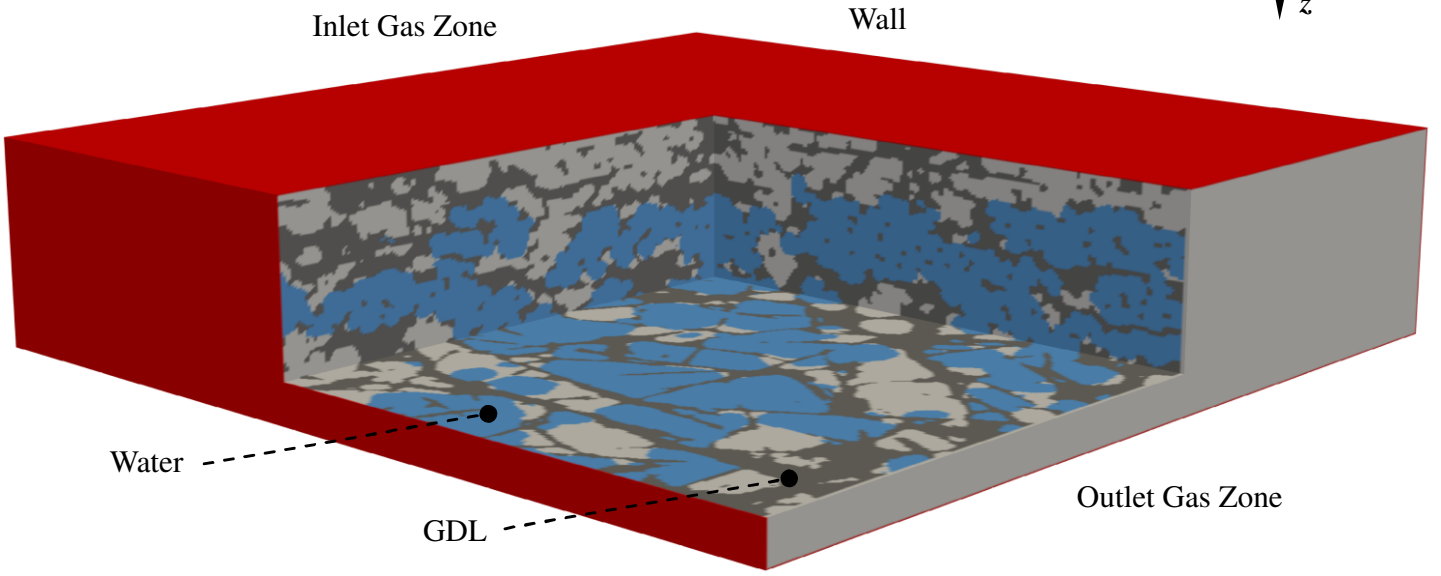

(b)

\section{In-Plane Direction}

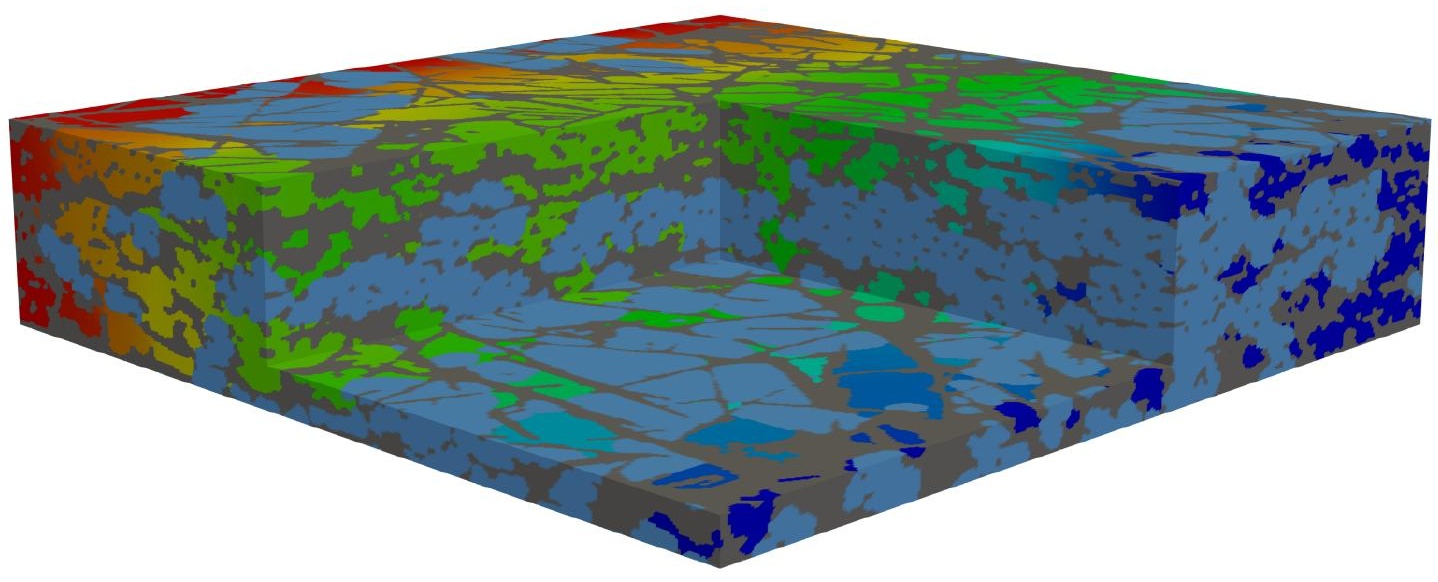

(c)

\section{Through-Plane Direction}

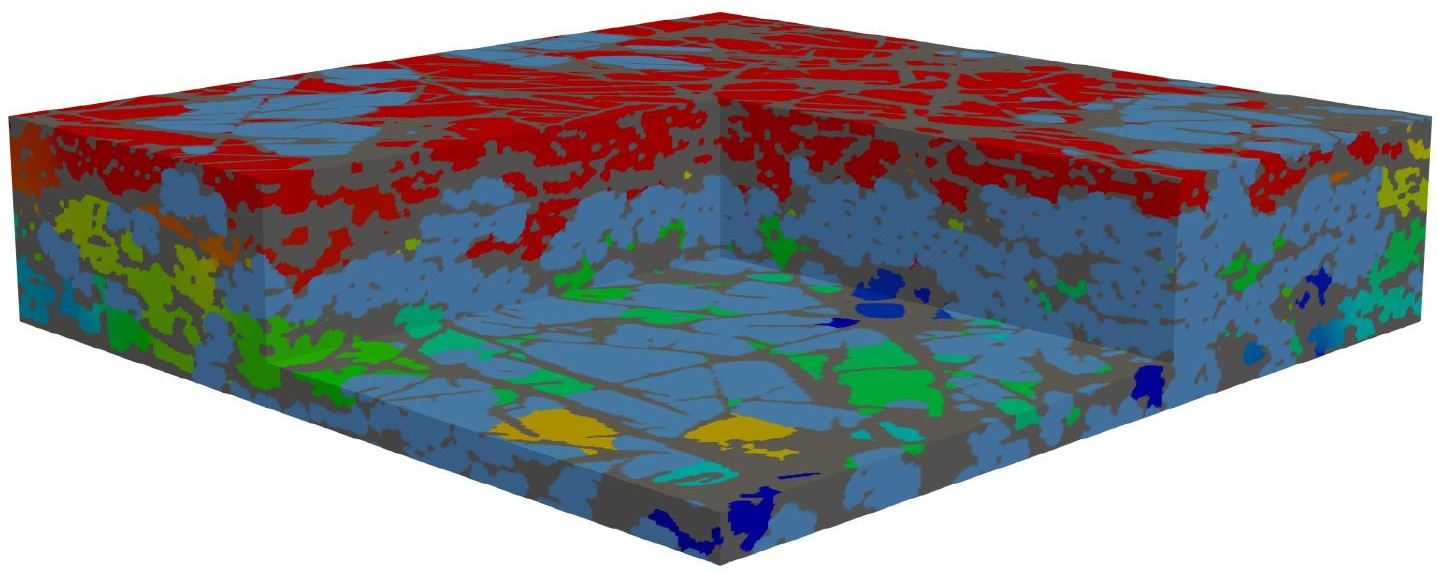


|Reduce Saturation Variation Across GDL

Reduce GDL Window Length $\delta_{\mathrm{z}}$ in Simulations

$$
\delta_{\mathrm{z}} / L_{\mathrm{z}}=100 \%
$$

(Full Thickness, FT)

$$
\delta_{\mathrm{z}} / L_{\mathrm{z}}=80 \%
$$

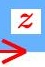



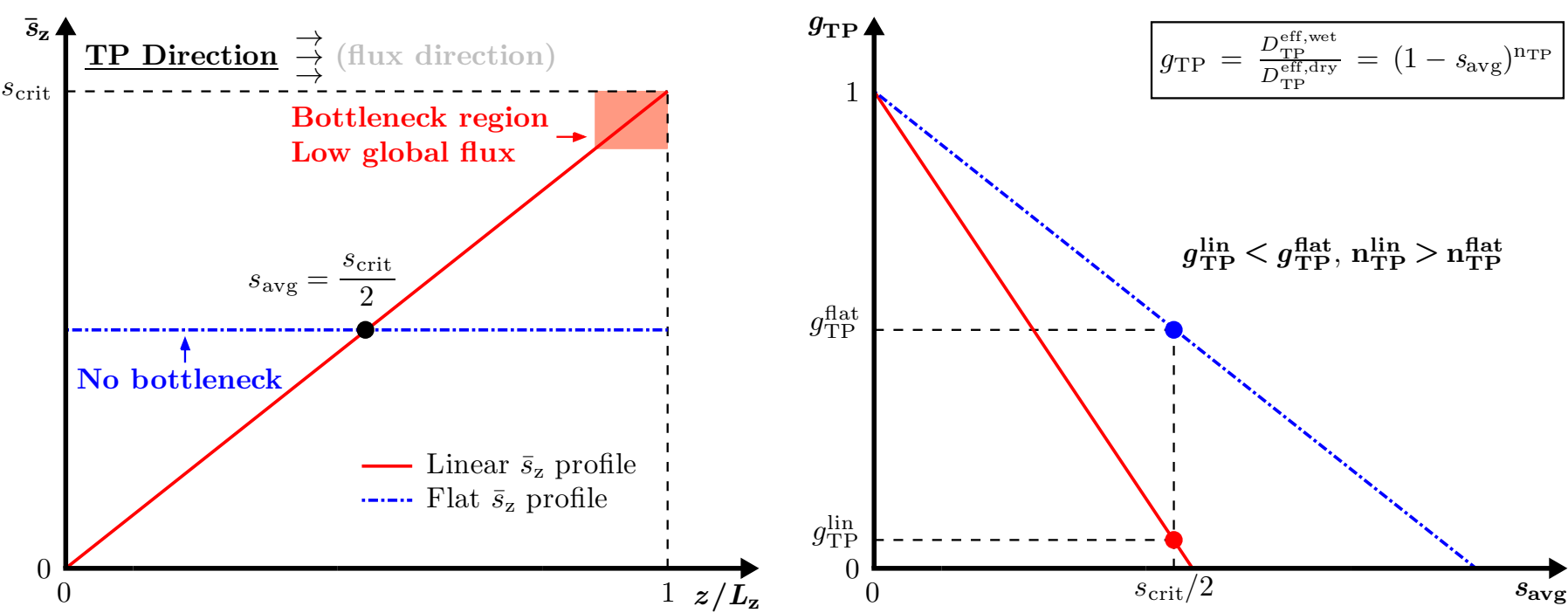

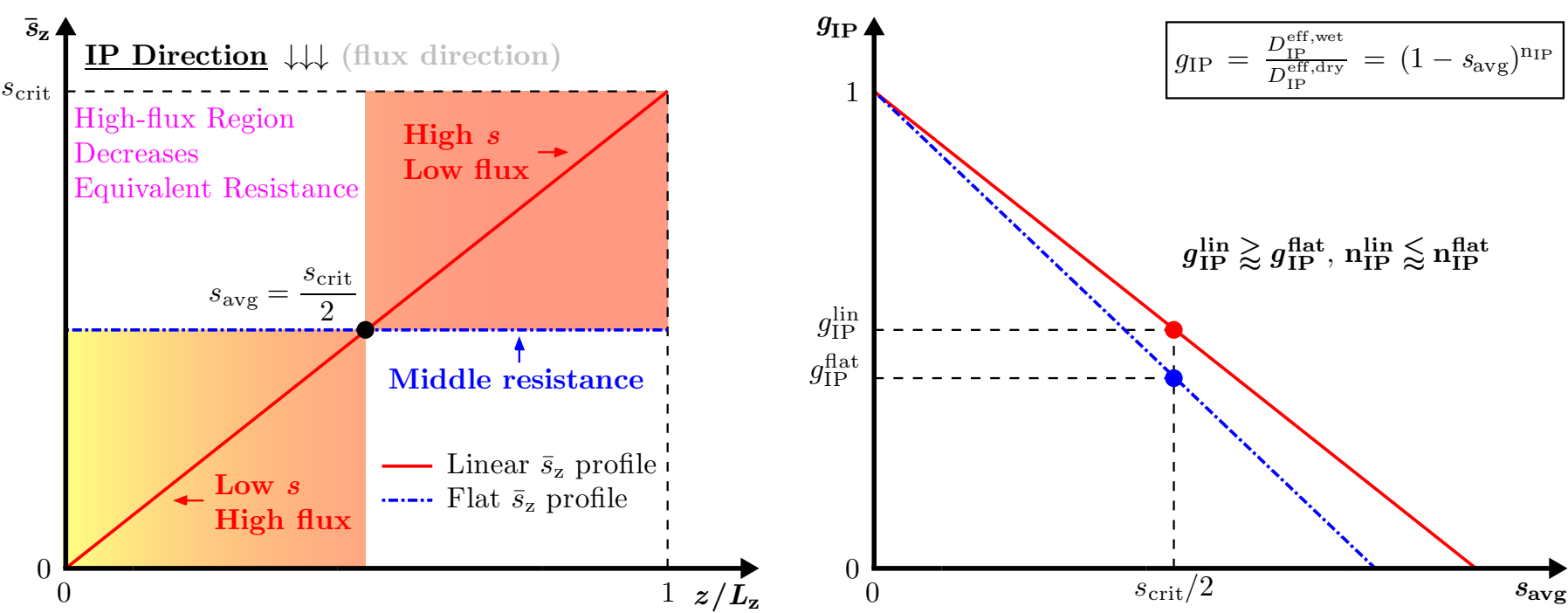


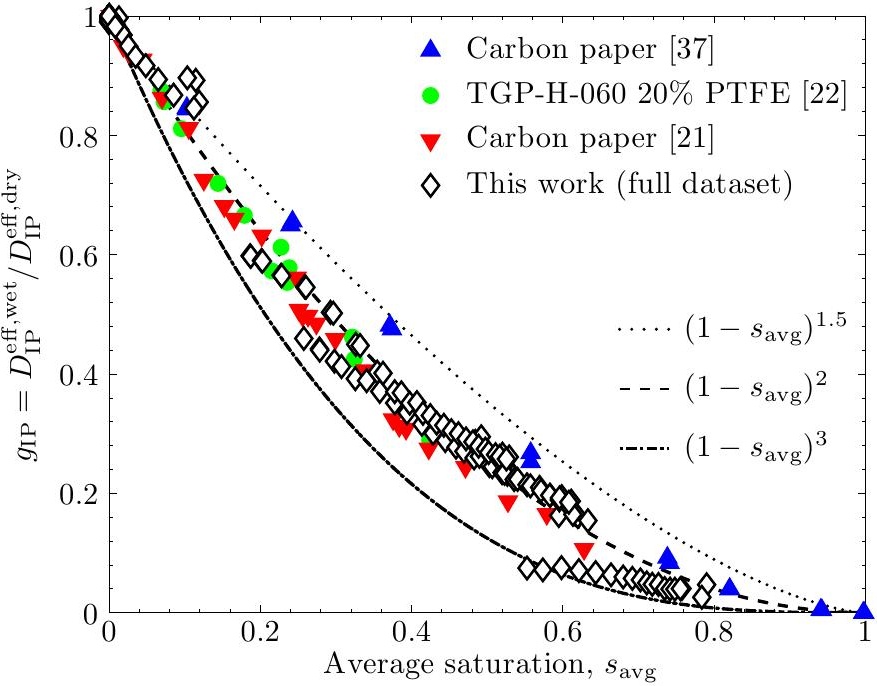


- Gas diffusion is simulated on X-ray images of partially-saturated carbon-fiber GDLs.

- The dry effective diffusivity is anisotropic and decreases by the addition of PTFE.

- Invading water has a dramatic influence on through-plane diffusive transport.

- The effective diffusivity in finite-thickness GDLs is not uniquely described by average saturation.

- The distribution of water through the GDL has major impacts on the effective diffusivity. 